\title{
Single-nuclei chromatin profiling of ventral midbrain reveals cell identity transcription factors and cell-type-specific gene regulatory variation
}

\author{
Yujuan Gui ${ }^{1}$, Kamil Grzyb², Mélanie H. Thomas², Jochen Ohnmacht ${ }^{1,2}$, Pierre Garcia², Manuel Buttini², \\ Alexander Skupin ${ }^{2}$, Thomas Sauter ${ }^{1}$ and Lasse Sinkkonen ${ }^{1 *}$ (D)
}

\begin{abstract}
Background: Cell types in ventral midbrain are involved in diseases with variable genetic susceptibility, such as Parkinson's disease and schizophrenia. Many genetic variants affect regulatory regions and alter gene expression in a cell-type-specific manner depending on the chromatin structure and accessibility.

Results: We report 20,658 single-nuclei chromatin accessibility profiles of ventral midbrain from two genetically and phenotypically distinct mouse strains. We distinguish ten cell types based on chromatin profiles and analysis of accessible regions controlling cell identity genes highlights cell-type-specific key transcription factors. Regulatory variation segregating the mouse strains manifests more on transcriptome than chromatin level. However, cell-type-level data reveals changes not captured at tissue level. To discover the scope and cell-type specificity of cis-acting variation in midbrain gene expression, we identify putative regulatory variants and show them to be enriched at differentially expressed loci. Finally, we find TCF7L2 to mediate trans-acting variation selectively in midbrain neurons.

Conclusions: Our data set provides an extensive resource to study gene regulation in mesencephalon and provides insights into control of cell identity in the midbrain and identifies cell-type-specific regulatory variation possibly underlying phenotypic and behavioural differences between mouse strains.
\end{abstract}

Keywords: Single-nuclei ATAC-seq, Mouse strains, Genetic variation, Midbrain, Cell-type identity, Wnt signalling

\section{Background}

The ventral midbrain, or mesencephalon, is one of the most evolutionary conserved brain structures in mammals [1]. It is involved in tasks such as processing of sensory information and eliciting motor and cognitive control through dopaminergic circuits [1]. It is of particular interest due to its involvement in human diseases, such as Parkinson's disease and schizophrenia, whose

*Correspondence: lasse.sinkkonen@uni.lu

${ }^{1}$ Department of Life Sciences and Medicine (DLSM), University of Luxembourg, Belvaux, Luxembourg

Full list of author information is available at the end of the article development and progression are significantly influenced by individual's genetic susceptibility [2-5].

Like other brain regions, midbrain harbours many different cell types that exhibit both functional and molecular diversity [6-8]. A cell type can be distinguished by its gene expression profile. Transcriptomic analysis at single cell level has identified 20 cell types and 58 subtypes in ventral midbrain [8]. These unique gene expression profiles defining cell state and cellular identity are controlled by epigenetic mechanisms and achieved by dynamic interplay between chromatin and expressed transcription factors (TFs). In particular, regulation by 
cell-type-specific master regulators, TFs that open and specifically bind to gene regulatory regions, results in distinct gene expression profiles between cell types [9]. The chromatin landscape and accessibility of TF binding sites can be elucidated using epigenomic analysis such as the assay for transposase-accessible chromatin followed by sequencing (ATAC-seq) [10]. So far, the ability to isolate pure populations of various brain cell types has been limiting the progress in the field. However, recent developments in single-nuclei chromatin assays have now enabled massive parallel analysis of cell-type-specific chromatin profiles in their native context [11-14].

Typical human genomes differ from each other on average by 5 million genetic variants [15]. Vast majority of these are located in the non-coding genome and those associated with complex traits are enriched at accessible gene regulatory regions in a cell-type-specific manner [16]. Genetic variation at regulatory regions can influence TF binding and thereby gene expression either in cis or in trans, hereafter referred to as gene regulatory variation [17]. Identifying genes, regulatory regions and cell types affected by regulatory variants can help to understand the molecular mechanisms underlying the trait in question. C57BL/6J and A/J are two genetically distinct inbred mouse strains often used in neurobiology and to study complex genetic traits. The two strains segregate by $\sim 6$ million variants, comparable to the genetic variation between typical human individuals, making them an interesting model system to understand the effects of regulatory genetic variation on the phenotypic expression of complex traits. Indeed, the two strains show genetic differences also in traits associated with midbrain function. For example, $\mathrm{A} / \mathrm{J}$ is more anxious and less social [18] and has lower motor activity [19]. We have recently shown that the two strains exhibit significant differences in their ventral midbrain transcriptomes [20], but the underlying gene regulatory changes, as well as the cell-type-specific epigenomic profiles of mouse ventral midbrain, are not known.

Here we performed chromatin accessibility profiling of mouse ventral midbrains from $\mathrm{C} 57 \mathrm{BL} / 6 \mathrm{~J}$ and $\mathrm{A} / \mathrm{J}$ at single-nuclei level (snATAC-seq). We identify $>260,000$ individual regulatory regions across 20,658 epigenomic profiles which can distinguish ten main cell types in ventral midbrain, and define sets of unique cell identity genes and identify TFs controlling their expression.
Comparison of gene expression and chromatin accessibility between the mouse strains shows that genetically driven differences are more striking at the transcriptomic than chromatin accessibility level. Nevertheless, regulatory regions with alternated chromatin accessibility are enriched at differentially expressed genes and can reveal cell-type-specific gene regulation. We find cis-acting variants to be enriched at differentially expressed genes and pinpoint the extent of cell-type-specific gene regulatory variation. Finally, we suggest canonical Wnt signalling to be a mediator of trans-acting variation in midbrain neurons.

\section{Results}

Single-nuclei chromatin profiles of ventral midbrain and identification of major cell types in two mouse strains

To unravel the cell-type-specific gene regulation in midbrain, and how it impacts genetic regulatory variation, we performed ATAC sequencing at single-nuclei level (snATAC-seq) on dissected midbrains from two genetically distinct mouse strains, C57BL/6J and A/J (Fig. 1, Additional file 1: Figure S1). Perfused ventral midbrain sections from two hemibrains of one mouse for both strains were used for the partitioning and barcoding with a total of 13,640 and 13,259 nuclei from C57BL/6J and A/J, respectively, and following high throughput sequencing. After filtering of multiplets and nuclei with low coverage, approximately 290 million reads per mouse strain were retained, corresponding to 10,298 (C57BL/6J) and 10,360 $(\mathrm{A} / \mathrm{J})$ individual accessibility profiles (Fig. 1A). The bulk chromatin accessibility profile aggregated across single nuclei (bulk snATAC-seq) from C57BL/6J showed a total of 231,390 peaks. Notably, $99.7 \%$ of regular bulk ATACseq peaks obtained from an independent C57BL/6J midbrain section overlapped with bulk snATAC-seq peaks (Additional file 1: Figure S1). Moreover, the bulk snATAC-seq profile from A/J with 235,157 peaks was also highly correlated with C57BL/6J profile (Pearson $R>0.97$ ). Finally, to distinguish accessible regions at enhancers and promoters actively engaged in transcriptional control, we performed ventral midbrain bulk level ChIP-seq analysis in both mouse strains for histone H3 lysine 27 acetylation (H3K27ac) [21, 22]. Both bulk ATAC-seq and H3K27ac ChIP-seq showed clear correlation with midbrain gene expression levels (Additional file 1: Figure S2).

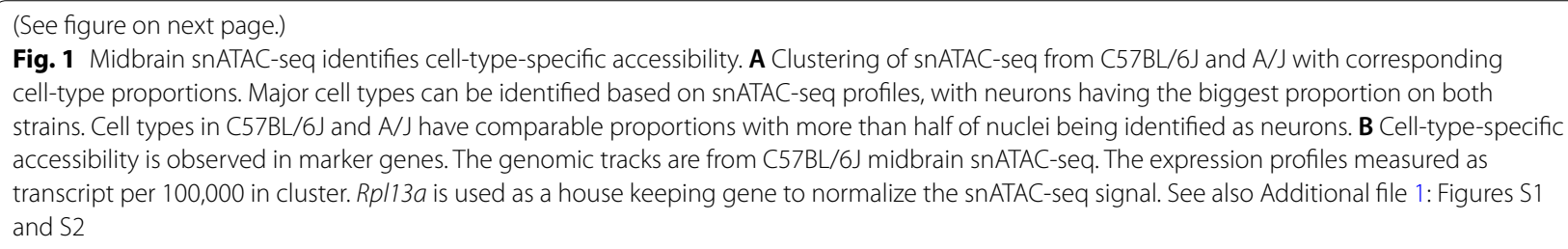




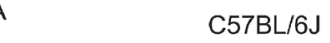

(Number of Nuclei: 10298)
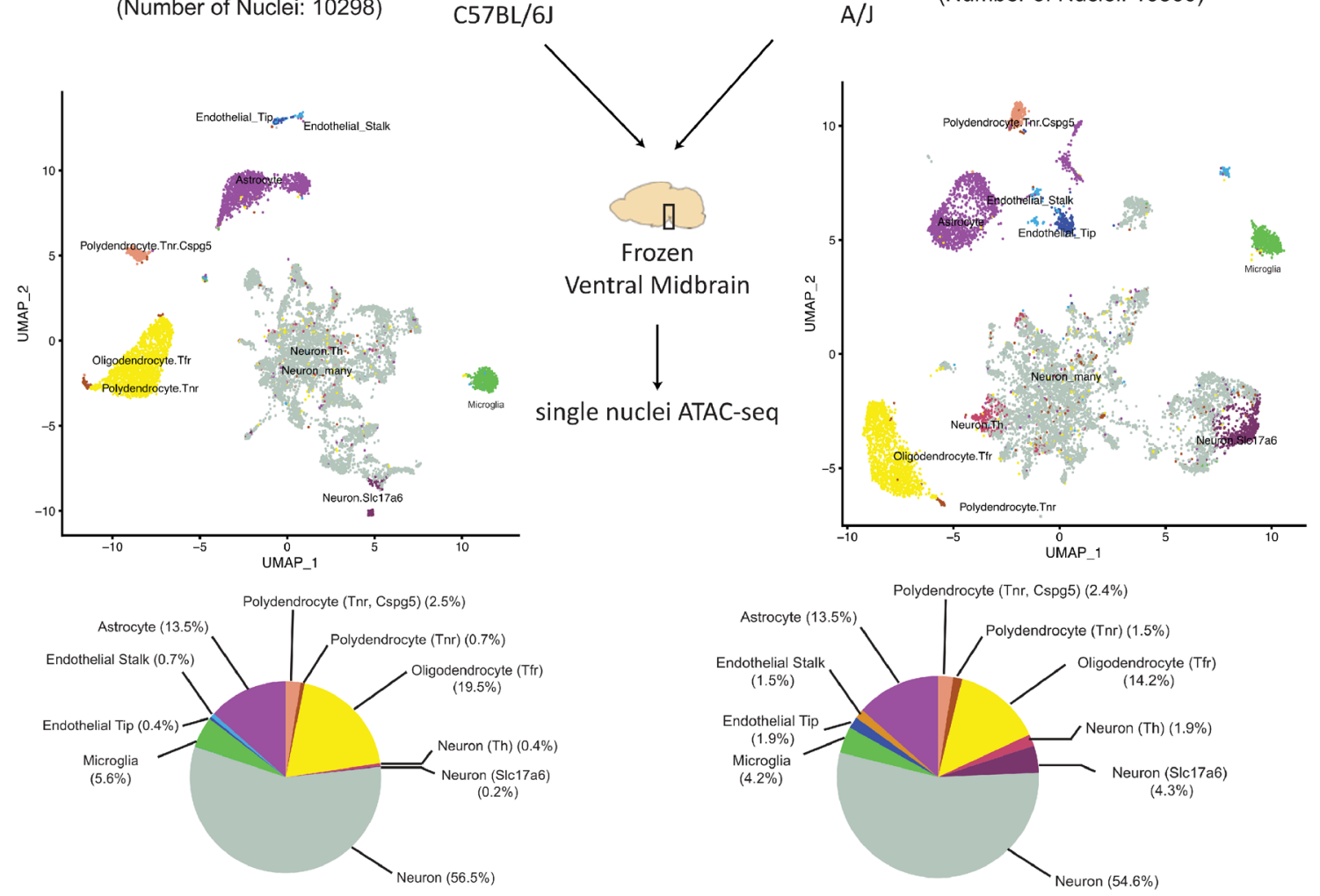

A/J
(Number of Nuclei: 10360)

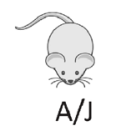

B

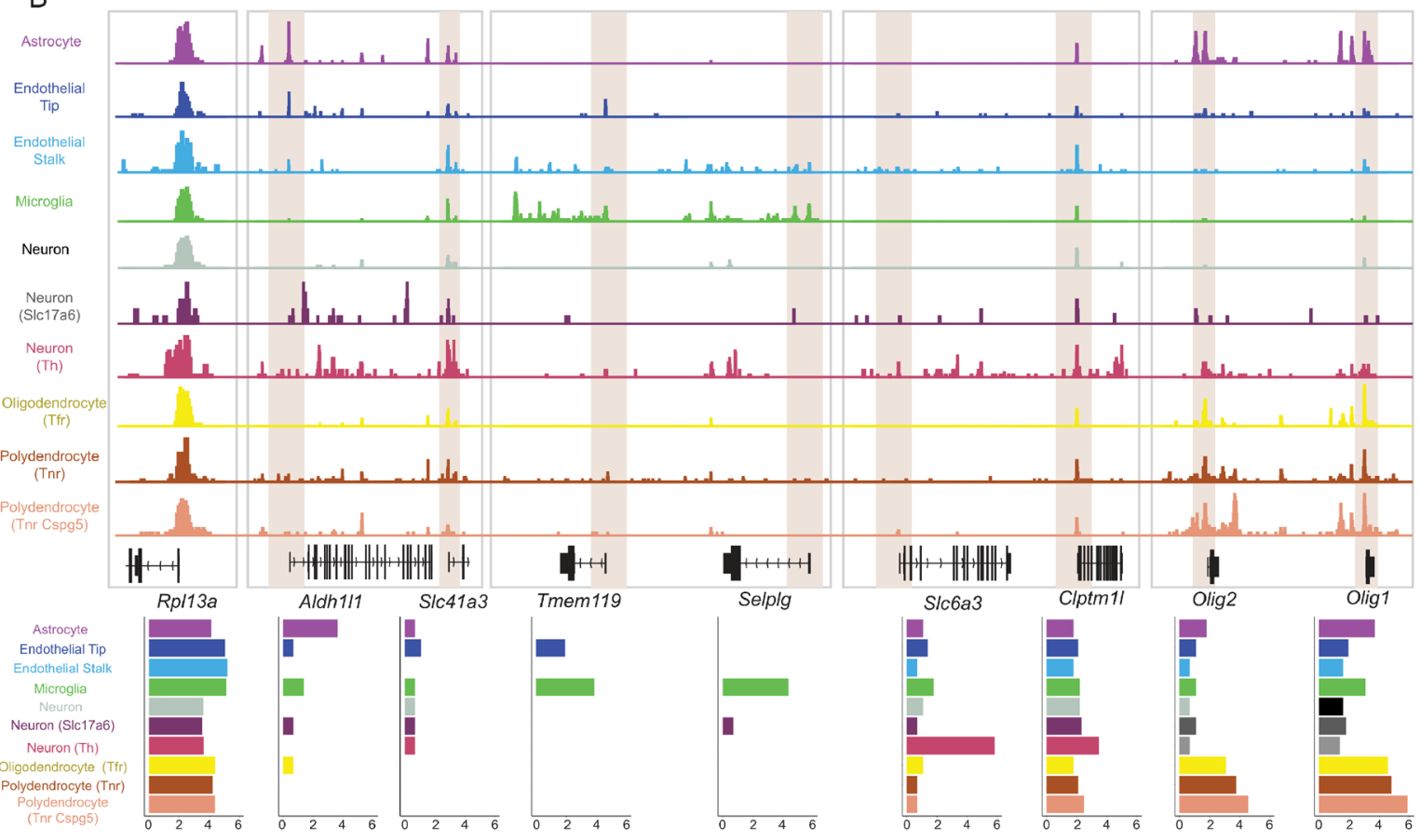

Transcript Per 100,000 in Cluster

Fig. 1 (See legend on previous page.) 
Using an existing single cell genomics toolkit [23, 24], the dimensionality of snATAC-seq was calculated by performing latent semantic indexing (LSI), to allow clustering of the cells with uniform manifold approximation and projection (UMAP) (Fig. 1A). A gene activity matrix of snATAC-seq was established by counting reads in the gene body and the promoter region $[2 \mathrm{~kb}$ upstream of transcription start site (TSS)]. To annotate the obtained clusters as individual cell types, we took advantage of existing single cell RNA sequencing (scRNA-seq) of mouse midbrain [8]. Through identification of anchor genes shared between the gene activity matrix of snATAC-seq and the highly variable features in scRNA-seq, we could identify 10 different midbrain cell types with distinct chromatin accessibility profiles and sufficient numbers of cells in both strains (Fig. 1A, Additional file 2: Table S1). The cell types are grouped into six main clusters consisting of glial cells, such as astrocytes (13.5\%), microglia (4.2-5.6\%), oligodendrocytes (14.-19.5\%), and two subtypes of polydendrocytes $\left(\mathrm{Tnr}^{+}\right.$ and $\mathrm{Tnr}^{+} / \mathrm{Cspg5}^{+}$) (3.2-3.9\%), two different types of endothelial cells (stalk and tip; 1.1-3.4\%), and the largest and most diffuse cluster neurons making up more than half of all cells (57.1-60.8\%). Although scRNA-seq data could distinguish up to 30 neuronal subtypes in midbrain through combinations of marker gene abundances [8], at chromatin accessibility level these could not be clearly distinguished. Instead, only three classes of neuronal cell types could be well distinguished: thalamus glutamatergic neurons (referred to as $S l c 17 a 6^{+}$neurons), dopaminergic neurons (referred to as $T^{+}$neurons), and a broader group of neurons consisting to large extent, but not exclusively, from different $\mathrm{Gad}^{+}$GABAergic neurons (referred to simply as neurons).

Interestingly, an increased proportion of $\mathrm{Th}^{+}$and Slc17a6 ${ }^{+}$neurons and decreased proportions of oligodendrocytes and macrophages could be detected in $\mathrm{A} / \mathrm{J}$ samples compared to C57BL/6J, while the proportion of astrocytes and $\mathrm{Tnr}^{+} / \mathrm{Cspg} 5^{+}$polydendrocytes remained almost identical (Fig. 1A).

Inspection of genomic loci encoding for known celltype-specific marker genes in C57BL/6J samples disclosed highly cell-type-selective chromatin accessibility that was well correlated with gene expression levels in scRNA-seq data of mouse midbrain (Fig. 1B). While ubiquitously expressed Rpl13a gene had high and consistent levels of accessibility across the cell types, known marker genes for astrocytes (Aldh1l1) and microglia (Tmem119 and Selplg) $[25,26]$ were expressed and most accessible in the respective cell types, especially at their TSS. Similarly, the gene encoding for dopamine transporter (Slc6a3) had highest levels of expression and accessibility in the $T h^{+}$ neurons, while in other neurons almost no signal could be detected. At the same time, the adjacent Clptm1l gene harboured an accessible promoter in all of the cell types. Finally, the locus encoding for two TFs required for oligodendrocyte generation and maturation, Olig1 and Olig2 $[27,28]$, showed highest accessibility in subtypes of polydendrocytes and oligodendrocytes, as well as astrocytes, again consistent with the gene expression levels.

Importantly, the accessibility profiles between C57BL/6J and A/J were highly comparable also at the level of individual cell types and could equally highlight cell-type-specific accessibility consistent with gene expression levels, as shown in Additional file 1: Figure S1 for Aif1, a known marker gene for microglia.

Taken together, our snATAC-seq profiling produced over 20,000 chromatin profiles of mouse midbrain cell types with comparable quality from two different mouse strains. These data allow the distinction of 10 different midbrain cell types at epigenomic level that are consistent with known gene expression profiles.

\section{Identification of cell identity genes and associated regulatory regions from single cell data}

To leverage the available data for the identification of TFs controlling cellular identity in adult midbrain cell types, we first set out to determine the genes whose expression was selective for each cell type. To obtain these cell identity genes, we used the existing scRNA-seq of the mouse midbrain, and for each gene determined the 85th percentile of its expression across all cell types indicating gene specific "high expression" (Fig. 2). To filter out genes being selectively expressed in a specific cell type, at least $60 \%$ of the cells of that cell type have expression not less than the 85th percentile. Furthermore, to ensure uniqueness, no other cell type was permitted to have the same gene among its top expressed genes (as defined by their 85 th percentile) in more than $40 \%$ of the cells. Through this approach we could define between 47 and 412 identity genes for each cell type (Fig. 2; Additional file 3: Table S2). On average, 170 genes per cell type were determined. To confirm the relevance of the genes to the biology of the cell type in question, GO enrichment analysis for biological processes was performed. In keeping with the genes' role in the molecular and biological identity of the cell types, the top enriched GO terms included: Positive regulation of angiogenesis for endothelial stalk cells; Neutrophil mediated immunity for microglia; Axonogenesis, Neurotransmitter transport, and Regulation of synaptic vesicle exocytosis for the different neurons; Septin ring assembly and Myelination for oligo- and polydendrocytes; and Negative regulation of neuron differentiation for astrocytes. Examples of gene expression profiles of identity genes from selected cell types are shown in 
Fig. 2B. Full list of enriched GO terms are provided in Additional file 4: Table S3.

Next, to determine gene regulatory regions controlling the expression of cell identity genes, we performed peak calling on cell-type-specific aggregate ATAC-seq signals and associated the peaks to the defined identity genes of the respective cell types using GREAT (basal regulatory region $\pm 100 \mathrm{~kb}$ from TSS or up to nearest gene [29]). This resulted in 100-1200 accessible regions likely to control cell identity gene expression in each cell type (Fig. 2; Additional file 5: Table S4).

\section{Cell-type-specific chromatin accessibility profiles uncover cell identity regulating transcription factors}

Comparison of the chromatin accessibility levels across the cell types confirmed a clear increase in accessibility at the obtained cell identity peaks associated with respective cell identity genes (Fig. 3A). The highest increase in signal over background of aggregated midbrain cells was always detected in the corresponding cell types expressing the associated identity genes. At the same time, depletion of signal could be detected in other cell types. Interestingly, the level of accessibility also reflected the developmental relationships of the cell types. The strongest depletion of signal could be detected in the developmentally most distant cell type, microglia [30]. Consistently, microglia identity peaks showed the strongest depletion in all other cell types. In contrast, neuron identity peaks showed no major depletion of signal in the related $T h^{+}$neurons and vice versa. Altogether, our approach could accurately identify cell-type-specific gene regulatory regions controlling cell-type identity.

To identify TFs binding the regulatory regions and controlling cell-type-identity genes, we performed TF binding motif analysis in sequences enriched at cell identity peaks (Fig. 3B). This analysis was done for eight cell types with the highest sequencing coverage. Importantly, the analysis highlighted motifs for several TFs previously shown to control the differentiation or identity of the respective cell types. These included SOX9 in astrocytes [31], SPI1 in microglia (also known as SFPI1 or PU.1 [32, 33]), SOX17 in endothelial stalk [34], and SOX10 and SOX8 in oligodendrocytes $[31,35]$. The most enriched motif across cell types was the shared binding site for CTCF and CTCFL, the sequence occurring at insulator regions, where CTCF mediates chromatin looping events [9], indicating the formation of cell-type-selective chromatin looping and topological domains at the loci of cell identity gene loci.

In addition, NFI-family motif was enriched in astrocytes and polydendrocytes, consistent with its reported role in the transition from neurogenesis to gliogenesis [36] and the requirement of NFIC for expression of astrocyte marker genes [37]. Motifs enriched in microglia included MAF motif which can be bound by MAFB, a TF recently shown to be important for maintenance of homeostasis in adult microglia [38]. Interestingly, RFXfamily motif was highly enriched in both astrocytes and neurons. Indeed, $R f x 1, R f x 3, R f x 4$, and $R f x 7$ are known to be expressed and to play a role in the brain [39], with $R f \times 4$ showing the strongest expression in astrocytes, while $R f x 3$ and $R f x 7$ are abundant in different neurons [8]. Thus, our data warrant further investigation of individual TFs in the RFX-family in the cellular identity of midbrain neurons and astrocytes.

Together with RFX family, another TF with enriched motif in neurons, ZNF740, also has been shown to localize at gene enhancers active specifically in differentiated human neuronal cell lines, further supporting the relevance of this prediction across species [40]. Finally, the motifs enriched uniquely in $T h^{+}$neurons included binding sites for KLF family TFs, MEF2 TFs, and ZBTB7 TFs (that share their core motif with WT1 and ZNF263) (Fig. 3B). From these particularly Klf9, Mef2a, Mef2d, and $Z b t b 7 c$ show high expression in $\mathrm{Th}^{+}$neurons [8], with $M e f 2 d$ exhibiting the most selective expression, an observation that could guide more detailed experiments into their role in dopaminergic neuron identity.

\section{Genetically driven chromatin accessibility changes reveal cell-type-specific gene expression changes}

We have recently shown that the midbrain phenotypic differences between C57BL/6J and $\mathrm{A} / \mathrm{J}$ (and associated behavioural changes) are accompanied by extensive gene regulatory variation [20]. Based on tissue-level bulk RNA-seq analysis of 12 independent mice per strain,

\footnotetext{
(See figure on next page.)

Fig. 2 Regions controlling cell-type identity can be defined by combining snATAC-seq and scRNA-seq. A Schematic workflow to define cell-type-specific signatures. Digital gene expression is obtained from DropViz. For each gene, the 85th percentile of its expression across all cell types was calculated. To define a gene as a cell-type-identity gene, at least $60 \%$ of the cells of a cell type should have expression more than the 85th percentile, while at the same time no other cell type was permitted to have the same gene among its top expressed genes (above the 85 th percentile) in more than $40 \%$ of the cells. Enrichment analysis with cell-type-identity genes found $\mathrm{GO}$ terms corresponding to cell-type characteristics. The cell-type-identity peaks are defined by peaks overlapping with the regulatory regions of cell-type-identity genes (basal region $\pm 100 \mathrm{~kb}$ until nearby genes). Subsequently, the enriched motifs in cell-type-identity peaks are detected. B Examples of identified cell-type-identity genes. The identified cell-type-identity genes for six major cell types show selective expression in the respective cell types when observing scRNA-seq data of the entire population of midbrain cells
} 


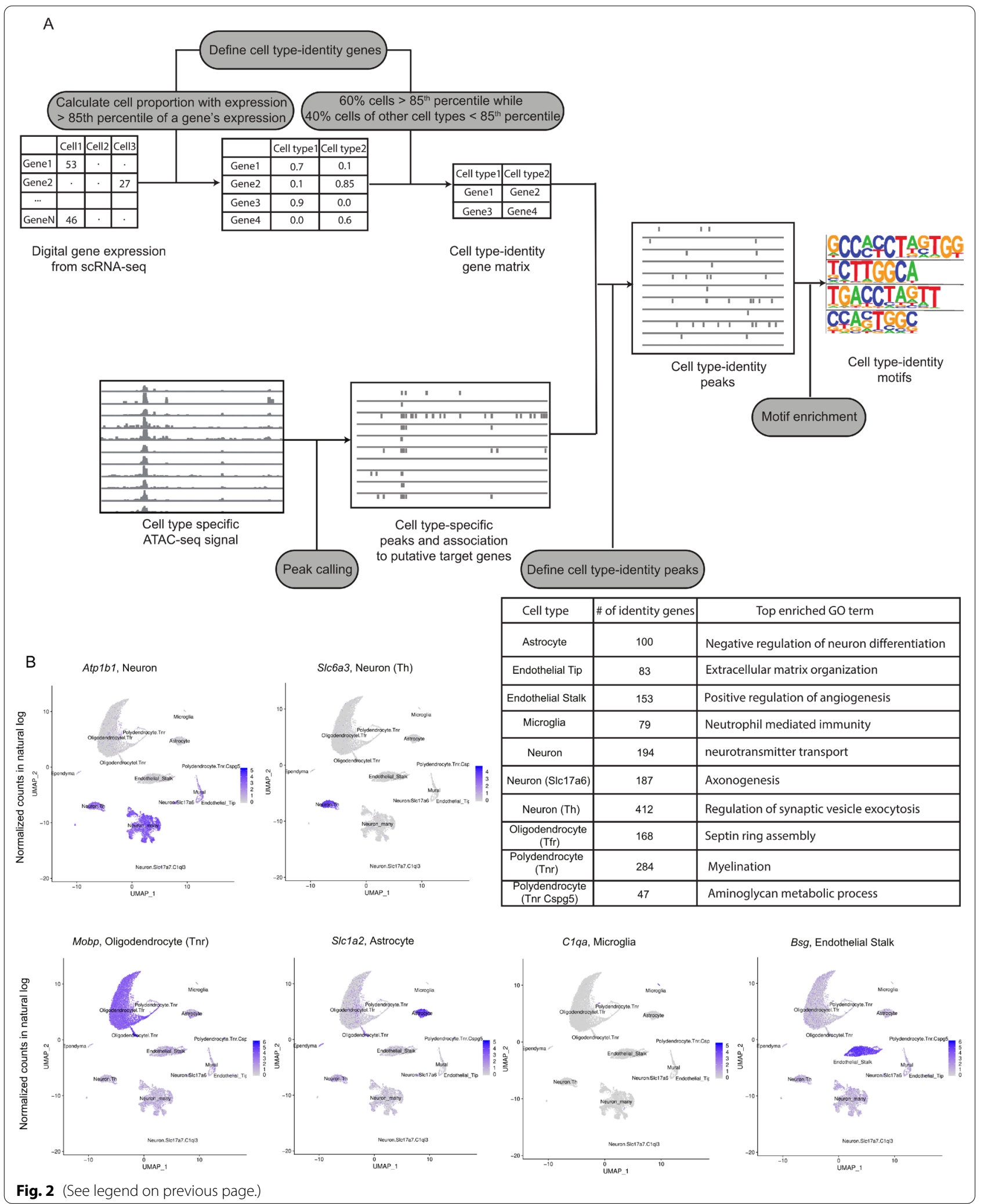




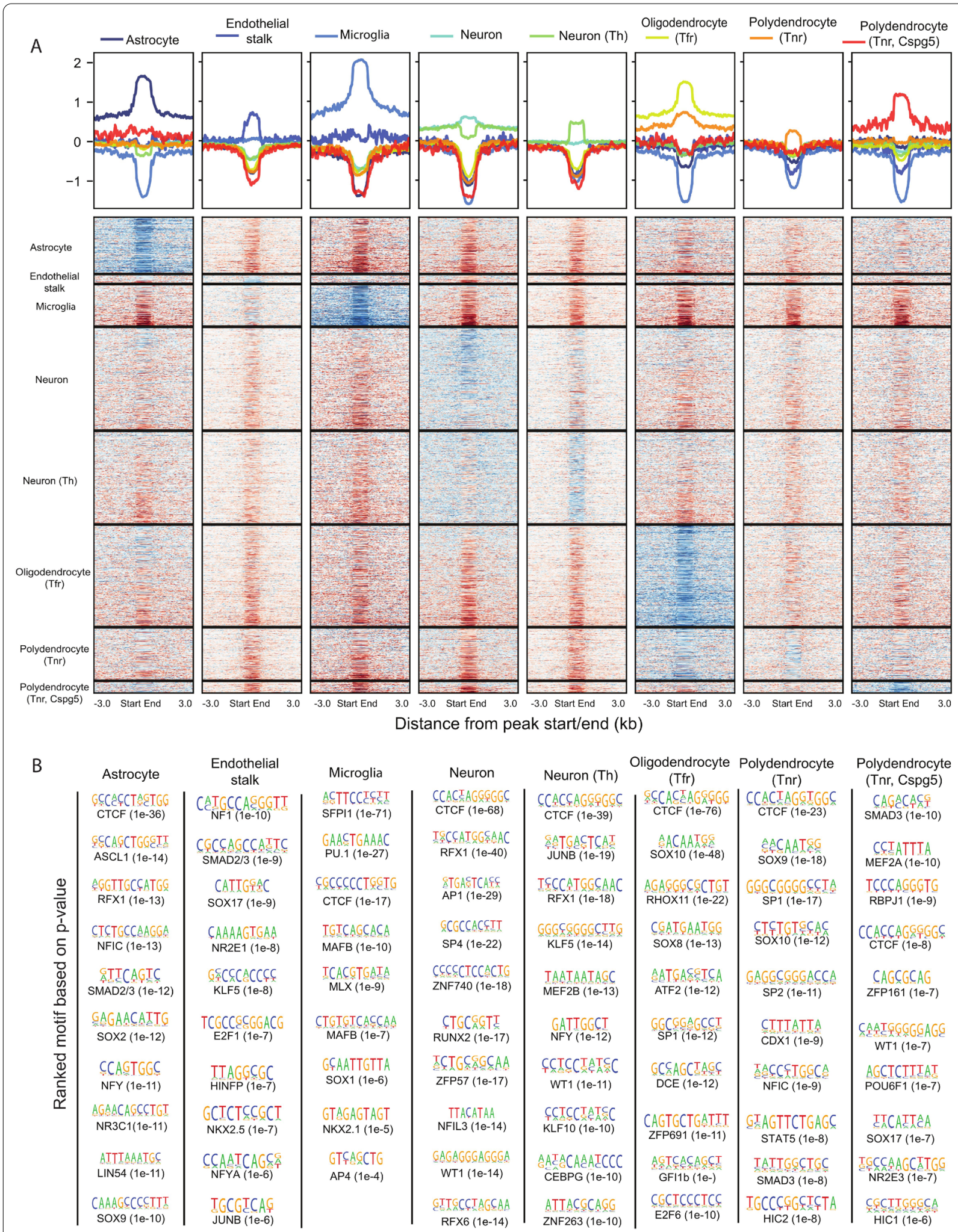

Fig. 3 Identification of cell-type-specific TFs controlling cellular identity. A Heatmap showing the enriched signal of cell-type-identity peaks in eight cell types. The analysis was done on C57BL/6J midbrain snATAC-seq. The background is constructed by merging the sampling reads (366,278 reads/ cell type) from each cell type. The raw signal is normalized to the background and library, following $\log _{2}$-transformation. The normalized signal is plotted $3 \mathrm{~kb}$ up- and downstream of peaks. B Motif enrichment analysis on cell-type-identity peaks. The PWM logos, names of the associated TFs and $p$ values are shown for each motif. The motifs are ranked according to $p$ values 
1151 genes are significantly differentially expressed ( $>$ twofold, FDR $<0.05$ ) in the ventral midbrains between the two strains (Fig. 4A). However, the cis- and transacting mechanisms underlying these genetically driven changes, and the affected cell types, are not known.

To address the contribution of chromatin level changes to the gene expression variation, we compared the bulk snATAC-seq signals from the mouse strains and focused on top differential peaks (FDR $<0.05$, top $1 \%$ of $\operatorname{logFC}$ ) (Fig. 4A). The extent of fold changes at the chromatin accessibility level was more modest than at the transcriptomic level. Nevertheless, 1317 of 263,709 called peaks were significantly altered at the bulk level. By associating accessible regions to their target genes, we could observe a significant enrichment of such regions at the differentially expressed genes. This indicates at least some of the gene expression changes could be linked to changes at the chromatin level. Observing the data at the level of individual cell type allowed detection of additional celltype-specific differentially accessible regions, indicating that cell-type-specific changes from rare cells could be masked by tissue level analysis. The significant enrichment of the differential peaks at differentially expressed genes held in all cell types except dopaminergic neurons which might result from their low sequencing coverage (Fig. 4A).

For genes, such as $I s o c 2 b$, the decreased gene expression in ventral midbrain of $\mathrm{A} / \mathrm{J}$ was accompanied by reduced accessibility of the promoter across all cell types (Fig. 4B). To confirm that the lost accessibility was also accompanied by reduced transcriptional activity, we observed H3K27ac levels at the promoter. Consistent with reduced ATAC-seq signal, H3K27ac was also lost at $I s o c 2 b$ locus in $\mathrm{A} / \mathrm{J}$.

For ubiquitously expressed genes such as $I s o c 2 b$ the altered gene expression could be associated with chromatin level changes even at bulk level analysis. However, for other genes, such as Olfr287, a reduced expression could be observed by RNA-seq although no signal was detectable at bulk chromatin level by any of the methods (bulk ATAC-seq, bulk snATAC-seq, and ChIP-seq). Still, when observing the cell-type-specific snATAC-seq data, an accessible region could be detected at $\mathrm{Olfr} 287$ promoter specifically in astrocytes. In addition, consistently with reduced gene expression, the chromatin was less accessible in $\mathrm{A} / \mathrm{J}$ (Fig. 4B).

In summary, gene regulatory variation in midbrain is associated with chromatin level changes in accessibility, although not at all loci and with lower sensitivity than in transcriptomic analysis. Interestingly, snATAC-seq can reveal cell-type-specific regulatory changes not captured in bulk level analysis.

\section{Putative cis-acting variants are enriched at midbrain regulatory regions of differentially expressed genes}

To obtain further insight into the mechanisms underlying the strain-specific gene expression, we next set out to address the extent of cis-acting regulatory variation contributing to the observed differences in the midbrain. For this we focused on identification of putative midbrain regulatory variants segregating $\mathrm{C} 57 \mathrm{BL} / 6 \mathrm{~J}$ and $\mathrm{A} / \mathrm{J}$. We first performed TF footprint identification on our midbrain chromatin accessibility profile obtained through the bulk ATAC-seq analysis. Then, these footprints were overlapped with $>6$ million variants segregating $\mathrm{C} 57 \mathrm{BL} / 6 \mathrm{~J}$ and $\mathrm{A} / \mathrm{J}$ to identify those with the potential to disrupt TF binding. Finally, the binding sites were overlapped with the midbrain H3K27ac profiles from both $\mathrm{C} 57 \mathrm{BL} / 6 \mathrm{~J}$ and $\mathrm{A} / \mathrm{J}$ to capture the binding sites engaged in transcriptional activity in either mouse strain, in total yielding 3909 putative regulatory variants of the ventral midbrain (Additional file 6: Table S5).

The capacity of the above approach to reduce the number of meaningful variants is illustrated in Fig. 5A with the examples of the Ddhd1, Zfp 619, and 4.5S ribosomal RNA ( $r R N A$ ) loci. Expression of $D d h d 1$, a gene coding for a phospholipase, is modestly but significantly reduced in A/J compared to C57BL/6J and shows accessible chromatin at its TSS and at an upstream enhancer site $>20 \mathrm{~kb}$ from the TSS. Both regions are marked by H3K27ac signals in both strains. One TF footprint could be identified at both the TSS and the distal enhancer, representing the putative TF binding sites controlling Ddhd1. From total of 603 variants at the $61 \mathrm{~kb}$ locus, only one coincides with an active TF binding site occupied in the midbrain, corresponding to a putative regulatory variant influencing Ddhd 1 expression in this brain region. Consistently, the affected enhancer shows decreased H3K27ac enrichment

(See figure on next page.)

Fig. 4 Association of differentially accessible regions with altered gene expression between C57BL/6J and A/J. A Differential peaks are highly associated with differential genes. Top differential peaks (labelled as red) are defined by Wilcoxon rank-sum test with FDR $<0.05$ within top $1 \%$ of logFC. The read counts in peaks of snATAC-seq bulk are $\log _{10}$-transformed. Peaks with low read count (less than median-1.5 median absolute deviation) are filtered out. To associate differential peaks to DEGs, peaks are overlapped with the regulatory region of DEGs (basal region $\pm 100 \mathrm{~kb}$ until nearby genes). As a control, random peaks are selected by bootstrapping with 1000 repetitions $(p<0.0099)$. The RPKM from bulk RNA-seq of $C 57 \mathrm{BL} / 6 \mathrm{~J}$ and $\mathrm{A} / \mathrm{J}\left(n=12\right.$ per strain) is also $\log _{10}$-transformed, and DEGs are defined as FDR $<0.05$ and $\log _{2}$-fold change $>1$ (labelled as red). B Cell-type-specific differential peaks correlate with gene expression in bulk RNA-seq. The differential peaks are labelled as green. ${ }^{*}$ FDR $<0.05$ 

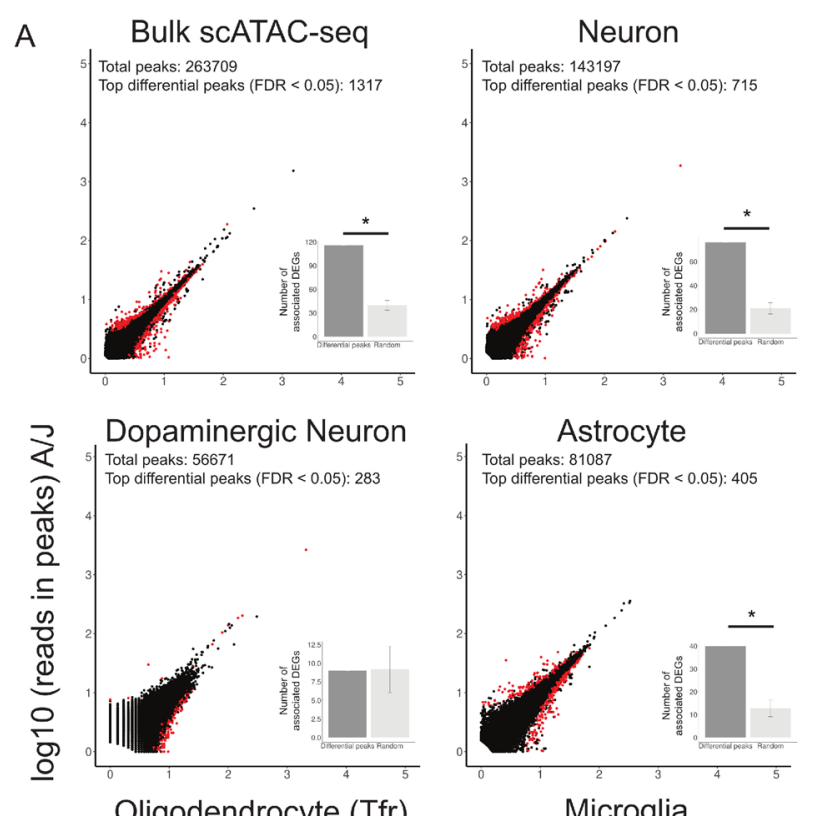

\section{Astrocyte}
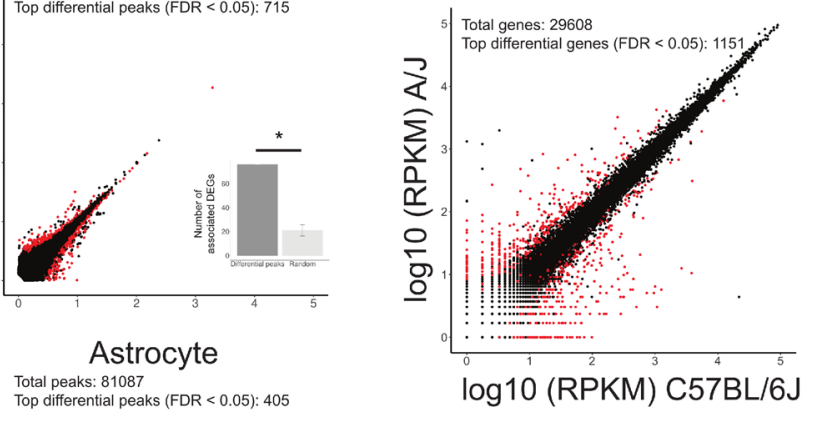

Oligodendrocyte (Tfr)

Total peaks: 81543
Top differential peaks (FDR < 0.05): 407

Top differential peaks (FDR < 0.05): 405
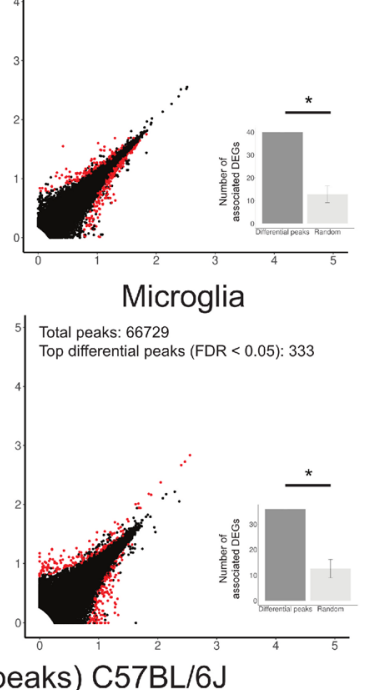

$\log 10$ (reads in peaks) C57BL/6J

B

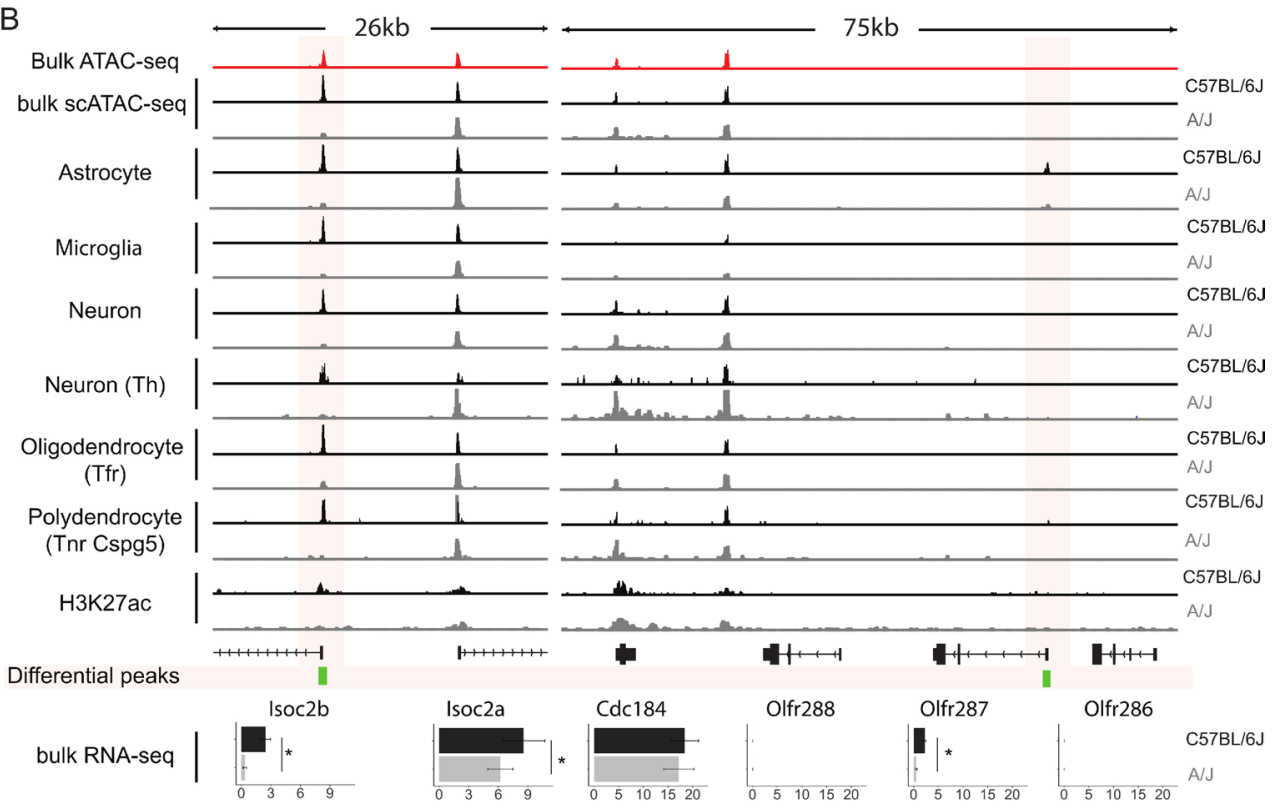

Fig. 4 (See legend on previous page.)

RPKM 
in the $\mathrm{A} / \mathrm{J}$. This illustrates how majority of genetic variants at any given locus are unlikely to affect gene expression and how, by focusing on those co-localizing within active regulatory regions, those most likely to act as regulatory variants can be identified.

If the midbrain gene regulatory differences between C57BL/6J and $\mathrm{A} / \mathrm{J}$ indeed depend on the cumulative effect of cis-acting variants, such as the variant at the Ddhd1 locus, the identified regulatory variants would be expected to be enriched in regulatory regions and TF binding sites at the differentially expressed gene loci compared to other expressed genes. To test this directly, we associated all putative regulatory variants to their likely target genes as already outlined in Fig. 2, and calculated the number of variants that on average associate with each of the 5082 differentially expressed genes $($ FDR $<0.05)$ (Additional file 7: Table S6) [20]. We did the same for all expressed genes found not to change between the strains (FDR $>0.05)$ and for an equal number of randomly selected expressed genes as controls. While unaffected genes and randomly selected genes were associated on average with 0.14 and 0.18 regulatory variants, respectively, this number significantly increased to 0.40 regulatory variants for the differentially expressed genes (Fig. 5B). Consequently, genetic variants located in midbrain regulatory regions do not show a random distribution but are instead enriched at the differentially expressed genes, suggesting they play an important role in explaining the observed transcriptomic differences.

Next, we considered whether localization of variants in the TF binding sites of active enhancers could also be associated directly with enhancer activity upstream of gene expression changes. With this aim we used THOR [41] to identify enhancer regions with significantly altered signal for the H3K27ac enhancer mark between midbrains of $\mathrm{C} 57 \mathrm{BL} / 6 \mathrm{~J}$ and $\mathrm{A} / \mathrm{J}$. Interestingly, 1126 of the 3909 putative regulatory variants localized within an enhancer region with differential H3K27ac enrichment under a stringent cutoff $\left(p<1 \times 10^{-18}\right)$ (Additional file 8: Table S7). This indicates that a large proportion of putative regulatory variants associate with enhancers that gain or lose activity between the mouse strains. For example, enhancer harbouring putative regulatory variants in the proximity of locus coding for $4.5 S$ rRNA, a ribosome-interacting non-coding RNA, exhibits a strong gain of enhancer activity in A/J compared to C57BL/6J (Fig. 5A). In addition, at locus of Zfp619, a gene coding for a zinc finger TF, both gain and loss of enhancer activity can be observed simultaneously at two separate enhancers associated with multiple putative regulatory variants. Taken together, disruption of TF binding by variants across thousands of enhancer regions is likely to alter enhancer activity, and thereby midbrain gene expression in genetically diverse mouse strains.

\section{Cell-type-specificity of cis-acting variants in the midbrain}

Having identified the putative cis-acting regulatory variants contributing to the midbrain gene expression phenotype between $\mathrm{C} 57 \mathrm{BL} / 6 \mathrm{~J}$ and $\mathrm{A} / \mathrm{J}$, we next sought to understand how cell-type-selective these variants are. Overlapping the putative regulatory variants with celltype-specific accessibility data suggested that majority of the variant binding sites (57.9\%) were accessible, with potential to affect gene expression, in at least 6 out of the 10 cell types (Fig. 5C). However, just under $14 \%$ of the variants were accessible in only 1 or 2 cell types, indicating non-coding variation can have cell-type-selective effects on gene expression (Fig. 5C). Indeed, variants with cell-type-selective accessibility in only $1-3$ cell types were significantly more often occurring at genes with altered expression than at other expressed genes (data not shown).

A number of regulatory variants were accessible specifically in neurons and associated with differentially expressed genes, representing neuron-specific gene regulatory variation. These include for example Tekt5, a gene expressed in excitatory neurons and upregulated in C57BL/6J (Fig. 5D). Despite the observed differential expression, Tekt 5 promoter appeared inaccessible in most cell types except for neurons of C57BL/6J, exactly at TSS overlapping with a putative regulatory variant within a TF binding site.

Taken together, while majority of cis-acting variants affect broad array of cell types, a large proportion can have cell-type-specific effects that cannot be dissected without single cell analysis.

\footnotetext{
(See figure on next page.)

Fig. 5 Putative regulatory variants are associated with differentially expressed genes and show cell-type-selective accessibility. A Examples of putative regulatory variants of $\mathrm{A} / \mathrm{J}$ found in the enhancer region upstream of $D d h d 1, Z f p 619$ and $R n 4.5$ s. The putative regulatory variants are defined as variants disrupting TF footprints located in active enhancers (defined by H3K27ac). B Each DEG $(5082, F D R<0.05)$ is associated with an average of 0.4 putative regulatory variants, while non-DEGs are associated with only 0.14 variants. Random: 5000 genes are randomly selected from all expressed genes. C Putative regulatory variants have differential accessibility across cell types. More than half of the variants are accessible in more than 6 cell types, while 7\% in only 1 cell type. D An example showing how putative regulatory variants with differential accessibility affect cell-type-specific gene expression. The variants locating near the TSS of Tekt5 are associated with TSS signal in neurons of C57BL/6J but not A/J, potentially resulting in upregulation of Tekt5 as shown in bulk RNA-seq. ${ }^{*} \mathrm{~F} R \mathrm{R}<0.05$
} 


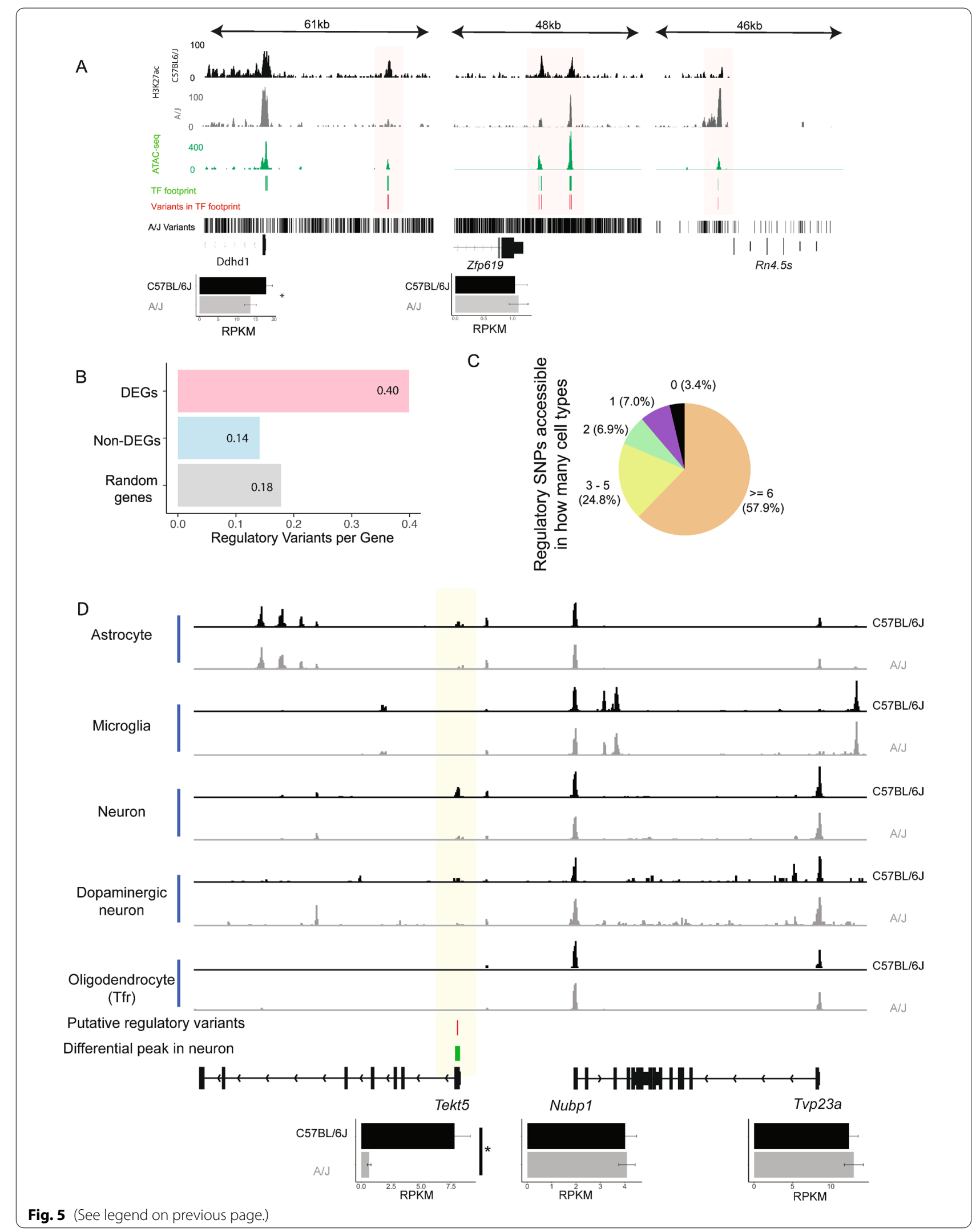


TCF7L2 as a mediator of trans-acting variation in midbrain neurons

A large fraction of midbrain gene expression variation could be linked to cis-acting regulatory variants, even with our strict criteria on the presence of variant in a TF footprint located in an active enhancer (Fig. 5A). Still, much differential gene expression remained unexplained. This could be due to cis-acting variants we have missed, but also due to trans-acting variants that can influence a number of target genes by altering a TF's activity, rather than its binding motif. A change in TF activity could be due to change in its expression level, but could also be due to alternations in other mechanisms controlling TF activity, such as post-translational modifications, protein-protein interactions or TF localization.

Genetic differences in non-dopaminergic neurons (such as $\mathrm{Gad2}^{+}$neurons), that make up much of our neuron population (Additional file 2: Table S1), have been suggested to contribute to strain specific behavioural differences, including anxiety, reward and motivation traits, such as ethanol consumption [42-45]. To identify mediators of trans-acting variation between $\mathrm{C} 57 \mathrm{BL} / 6 \mathrm{~J}$ and A/J in neurons, we performed motif enrichment analysis on 715 regions in neurons showing differential chromatin accessibility between the mice (Fig. 4A). This revealed the binding motif of TCF/LEF family, downstream TFs of the canonical Wnt signalling pathway [46], to be among the most enriched sequences found at the differentially accessible regions (Fig. 6A; $p=1.66 \mathrm{e}-14$ ). The enrichment was specific for Gad2 $2^{+}$neurons and could not be found in any other cell types (Additional file 1: Figure S3) or in the motif enrichment analysis for cell identity genes (Fig. 3). LEF1, TCF7L1, and TCF7L2 bind to the same DNA sequence but have often opposing or celltype-specific functions [47]. Inspecting chromatin accessibility across the cell types for the differential binding sites carrying the TCF/LEF motif revealed an increased signal specifically in the neurons (Fig. 6B). To determine which factor is expressed in the midbrain neurons and could mediate the observed enrichment and altered accessibility, we visualized their expression using scRNAseq data. Lef1 expression was limited to the endothelial cells (Fig. 6C) and Tcf7l1 showed only low or no expression across the cell types (Fig. 6D). However, Tcf7l2 had high expression in the $\mathrm{Gad}^{+}$and $S l c 17 a 6^{+}$neurons and polydendrocytes, showing a clear overlap with cells enriched for the respective binding motif (Fig. 6E). Consistently, an analysis of putative upstream regulators explaining the transcriptomic changes from RNA-seq of the mouse strains using Ingenuity Pathway Analysis (IPA) predicted TCF7L2 (also known as TCF4) and CTNNB1 (beta-catenin, binding partner of TCF7L2) to be among the top regulators based on the predicted activation score (Additional file 9: Table S8). Thus, the transcriptional activity of TCF7L2 is likely to be altered between $\mathrm{C} 57 \mathrm{BL} / 6 \mathrm{~J}$ and $\mathrm{A} / \mathrm{J}$ mice in the midbrain neurons.

Taken together, snATAC-seq analysis of tissues from genetically different strains can guide the elucidation of cell-type-specific impact of trans-acting variants and suggests neuron-specific differences in the canonical Wnt signalling pathway between two commonly used inbred mouse strains.

\section{Discussion}

Here we investigated the chromatin accessibility of cell types in mouse ventral midbrain in two different genetic backgrounds and provided a large resource of 20,658 single-nuclei chromatin profiles on 10 different cell types. This data set will benefit future studies on the role of these cell types in various processes involving ventral midbrain, such as movement control, cognition and reward mechanisms. A better understanding of midbrain cell types can also profit research on diseases, such as PD and schizophrenia. In particular, improved comprehension of genetic variation in gene regulation and how it impacts specific cell types, will pave the way for better prediction of genetic susceptibility and affected disease mechanisms.

Our findings on gene accessibility profiles and cell-type composition of the midbrain are consistent with existing knowledge from single cell transcriptomics [8]. However, neuronal subtypes are more difficult to distinguish at chromatin level than what has been achieved by transcriptomic analysis. Neuron subtypes clustered largely together, often with undefined borders between the subtypes (Fig. 1A). This result is expected. While chromatin accessibility is generally known to show positive correlation with gene expression $[48,49]$, and this is also true for our data (Additional file 1: Figure S2), enhancer accessibility does not necessarily reflect gene regulatory activity [50]. Indeed, chromatin accessibility profiles of cell types executing similar functions can be highly similar despite showing different expression patterns and being controlled by different master TFs $[16,51]$. Moreover, accessibility can signify priming of a locus for expression without commencing transcription $[52,53]$.

Nevertheless, we could reliably distinguish 10 of the 20 known midbrain cell types at the chromatin level, with eight cell types containing sufficient cells for detailed analysis (Fig. 3). Using the information about gene regulatory regions selectively associated with genes underlying cellular identity, we were able to predict the key regulators of each cell type through motif enrichment analysis. These included many factors previously determined to be necessary for the differentiation or the maintenance of the respective cell 


\begin{tabular}{|c|c|c|c|}
\hline A & f enrich & tt on neuron diffe & tial peaks \\
\hline & TF & Motif logo & q-value \\
\hline$\frac{d}{\pi}$ & NFYA & AAAGCTGACTGG & $1.39 \mathrm{e}-14$ \\
\hline 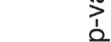 & TCF/LEF & GATCAAAGGT & $1.66 \mathrm{e}-14$ \\
\hline ह & RFX1 & IECCATGGCAAC & $1.70 \mathrm{e}-14$ \\
\hline 5 & RFXDC2 & GGTTGCTAGGCA & $7.12 \mathrm{e}-14$ \\
\hline & ZNF143 & GCATTCTGGG & $4.77 \mathrm{e}-$ \\
\hline
\end{tabular}

C

Lef1 Expression

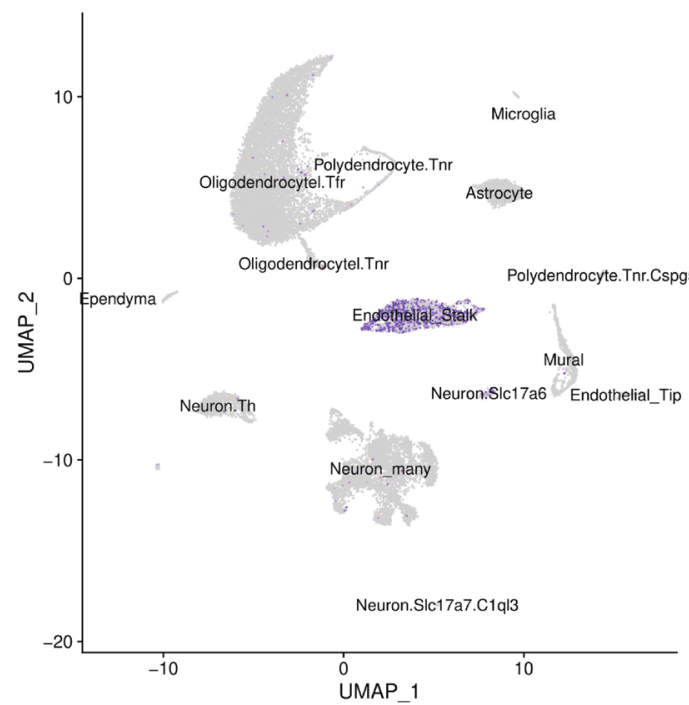

E

$$
\text { Tcf7/2 Expression }
$$

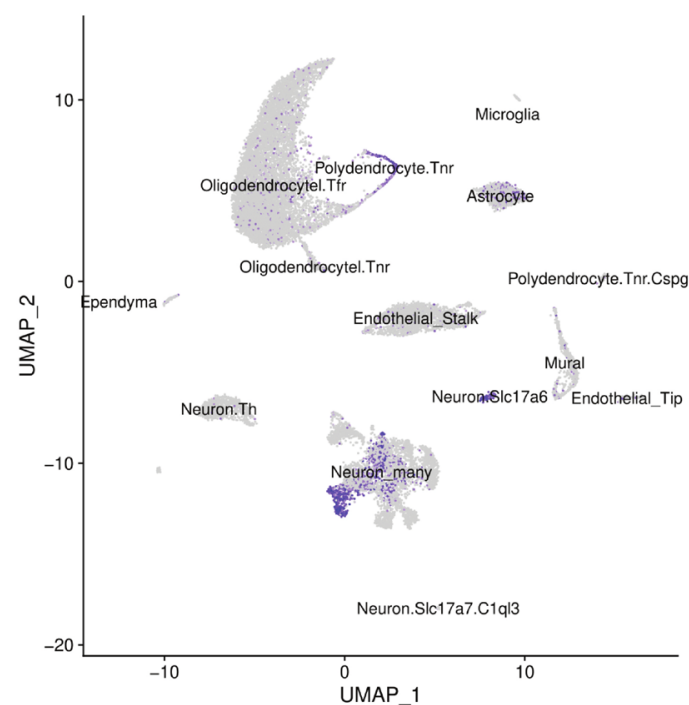

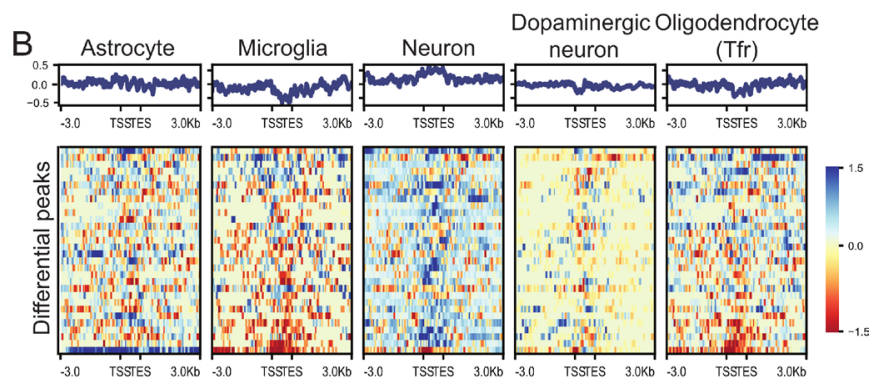

D

Tcf7/1 Expression

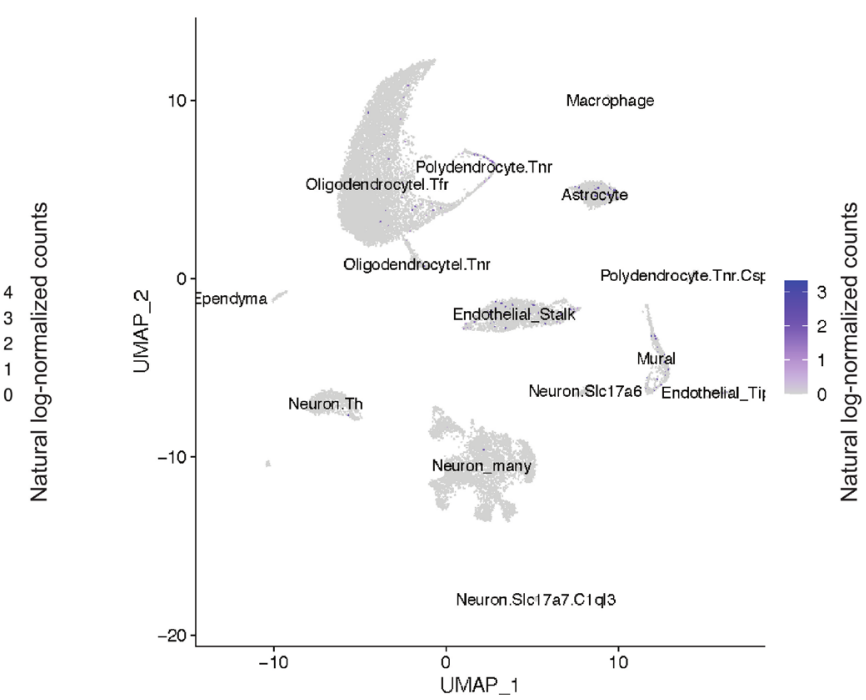

Fig. 6 TCF/LEF family as a mediator for trans-acting variation in neurons. A Motif enrichment analysis for regions of differential accessibility between C57BL/6J and A/J in neurons. B, C Regions of differential accessibility with motif for TCF/LEF family show increased accessibility in neurons compared to other cell types. D-E Lef1 is highly expressed in endothelial stalk, Tcf7/1 shows low expression in all cell types, while Tcf7/2 is abundant in neurons and polydendrocytes. See also Additional file 1: Figure S3 
state [31-33, 35, 54]. Among other insights, the results reveal a cell-type-specific role for CTCF binding sites in cellular identity and predict specific roles for MEF2, KLF, and ZBTB7 family TFs in dopaminergic neurons. Moreover, the results give additional support for a more detailed analysis of RFX family of TFs in regulation of neuro- and gliogenesis balance in the midbrain, something that is consistent with previously identified co-occupancy of RFX factors at SOX2-bound enhancers in neurodevelopment [55].

Genetic variation is known to drive gene expression changes that can trigger phenotypic differences [56-59]. We have recently shown over thousand genes to be differentially expressed in the mouse midbrain due to genetic variation between $\mathrm{C} 57 \mathrm{BL} / 6 \mathrm{~J}$ and $\mathrm{A} / \mathrm{J}$ [20]. Here we leveraged our single cell chromatin accessibility profiles to investigate the mechanisms, cell-type specificity, and extent to which this variation is reflected at the level of chromatin. Interestingly, the transcriptomic differences did not show a linear relationship with midbrain chromatin accessibility (Fig. 4A). The extent of change in accessibility was not comparable to mRNA level change either at bulk aggregate levels or in individual cell types. This is most likely reflecting the observation that TF binding and activity can alter gene expression without change in accessibility, for example if the locus is already open [60]. Still, when changes in accessibility did occur, this was often associated with local gene expression change. Importantly, some of the chromatin accessibility changes associated with differential gene expression could not be detected at all in tissue level ATAC-seq or ChIP-seq analysis despite high sequencing depth (Fig. 4B). Therefore, the improved resolution offered by single cell analysis allows further insights into regulatory interactions that could be missed in tissue level analysis.

Cis-acting variants that influence gene expression have been suggested to explain a significant part of the missing heritability [61-63]. Increasing number of such variants associated with human traits and diseases have now been experimentally validated [17]. By combining genetic information with ATAC-seq and ChIP-seq analysis, we found 3909 of the $>6$ million variants segregating C57BL/6J and A/J to localize in a TF binding site within an active and accessible enhancer in the ventral midbrain (Fig. 5). This number is consistent with the previous estimates that one in thousand mouse variants cause cis-regulatory effects [64]. Importantly, we found the putative cis-acting variants to be enriched at differentially expressed genes, indicating they do contribute to the gene expression phenotype.

A large proportion of the putative cis-acting variants showed cell-type-selective accessibility (Fig. 5B). This finding is consistent with previous work on impact of cis-acting variants on disease-associated regulatory variation in the major cell populations of human brain [65]. Combining the information on cell-type-specific impact of variants on gene regulation with genome-wide association studies and quantitative trait locus mapping for specific traits or diseases can provide insights on how the variants translate into phenotypes. $\mathrm{C} 57 \mathrm{BL} / 6 \mathrm{~J}$ and $\mathrm{A} / \mathrm{J}$ differ from each other for numerous phenotypes [66]. These include fear-conditioning and reward-related behaviours, such as ethanol consumption that are associated with midbrain dopamine signalling [43-45]. Combining our data on cell-type selectivity of cis-acting variants with genome-wide association studies of such phenotypes could reveal new connections between genes and the underlying mechanisms.

Besides cis-acting variation, differential gene expression and altered chromatin accessibility can also be mediated through trans-acting variation. Trans-acting variants can have many different mechanisms of action and the majority of genetically driven gene expression variation has been suggested to be due to trans-acting variation, often affecting hundreds or thousands of genes in a celltype-specific manner [62, 67, 68]. Dopaminergic neurons in ventral tegmental area of the midbrain contribute to phenotypes, such as reward-behaviour and fear [42]. Moreover, the associated GABAergic and glutamatergic neurons in midbrain can also control such behaviours, either together with or independent of dopamine signalling. With this in mind, we asked whether specific signature of trans-acting regulatory variation could be found in neurons between our strains that show differential behaviour, based on motif enrichment analysis at regions of differential accessibility (Fig. 6). We found an enrichment for the TCF/LEF binding motif, corresponding to the Wnt signalling response element recognized by their high mobility group (HMG) DNA-binding domain [46]. This suggests that Wnt signalling is altered between $\mathrm{C} 57 \mathrm{BL} / 6 \mathrm{~J}$ and $\mathrm{A} / \mathrm{J}$ in neurons. Based on scRNA-seq data, TCF7L2, but not LEF1 or TCF7L1, is abundantly expressed in midbrain $\mathrm{Gad2}^{+}$and $\mathrm{Slc} 17 a 6^{+}$neurons, indicating that alterations in chromatin accessibility and gene expression are due to changes in Wnt signalling and likely to be mediated by TCF7L2 (Fig. 6). Indeed, pathway analysis of the differentially expressed genes highlighted an enrichment of known Wnt target genes among the DEGs (Additional file 9: Table S8).

Involvement of TCF7L2 as the putative effector of the altered signalling in neurons is particularly intriguing, since TCF $7 L 2$ locus has been genetically associated with mental disorders, such as schizophrenia and bipolar disorder in humans $[69,70]$. Moreover, transgenic mice have revealed a dose-dependent role of $T c f 7 l 2$ in fearconditioning and anxiety, traits for which $\mathrm{A} / \mathrm{J}$ is known to 
significantly differ from C57BL/6J [43, 44]. Interestingly, the impact of $T c f 7 l 2$ dose on a number of phenotypes, including behavioural phenotypes, is known to depend on the genetic background of the mouse [71]. While some phenotypes induced by reduced $T c f 7 l 2$ expression can be observed in $\mathrm{A} / \mathrm{J}$ but not in C57BL/6J background, the opposite is true for several other phenotypes. Based on our data, one possible explanation for this could be the role of $T c f 7 l 2$ as a mediator of trans-acting variation, and thereby differential baseline activity of the Wnt pathway between the mouse strains. Understanding how Wnt signalling activity is altered between the mouse strains requires further analysis. However, it is interesting to note that expression of $W n t 2 b$, upstream ligand of Wnt pathway also genetically associated with bipolar disorder [69], is significantly decreased in the ventral midbrain of A/J compared to C57BL/6J [20].

\section{Conclusions}

Taken together, our single-nuclei chromatin analysis provides novel insights into transcriptional control of ventral midbrain cell types and a rich resource for further analysis of cellular identity and gene regulatory variation in this disease-relevant brain region.

\section{Methods}

\section{Animals}

All experimental procedures in this study were in compliance with the European Communities Council Directive 2010/63/EU, following the 3 Rs' requirements for Animal Welfare. We used two mouse strains in this study, C57BL/6J and A/J, purchased, respectively, from Charles River and Jackson Laboratory. The study cohorts were bred in-house at the Animal Facility of University of Luxembourg (Esch-sur-Alzette, Luxembourg) and the protocol was approved by the Animal Experimentation Ethics Committee (AEEC) according to the national guidelines of the animal welfare law in Luxembourg (Règlement grand-ducal adopted on January 11th, 2013). All mice were housed with a 12-h light-dark schedule and had free access to food and water.

For each mouse, intracardiac perfusion with PBS was performed after anesthesia with a ketamine-medetomidine mix (150 and $1 \mathrm{mg} / \mathrm{kg}$, respectively). The brain was extracted and both midbrains of each mouse were dissected, immediately snap-frozen, stored at $-80{ }^{\circ} \mathrm{C}$, and used for single cell partitioning as described below.

\section{Nuclei isolation}

For bulk ATAC-seq, frozen midbrains were minced in a Dounce Tissue Grinder (Sigma, D8939-1SET) with A pestle $\sim 10$ times following B pestle $\sim 20$ times in lysis buffer $\left[5 \mathrm{mM} \mathrm{CaCl}_{2}\right.$ (Merck, A546282), $3 \mathrm{mM}$ $\mathrm{Mg}(\mathrm{Ac})_{2}$ (Roth, P026.1), $10 \mathrm{mM}$ Tris $\mathrm{pH} 7.8,0.17 \mathrm{mM}$ $\beta$-mercaptoethanol (Gibco, 21985-023), $160 \mathrm{mM}$ sucrose (Sigma, S0389), 0.05 mM EDTA, 0.05\% NP40 (Sigma, I3021)]. The lysate was layered on $3 \mathrm{~mL}$ sucrose cushion [1.8M sucrose, $3 \mathrm{mM} \mathrm{Mg}(\mathrm{Ac}) 2,10 \mathrm{mM}$ Tris $\mathrm{pH} 8.0$, $0.167 \mathrm{mM} \beta$-mercaptoethanol], following ultra-centrifugation 30,000g for $1 \mathrm{~h}$ (Rotor:Beckman Coulter, MLS50) at $4{ }^{\circ} \mathrm{C}$. After centrifugation, the supernatant was discarded and the nuclei pellet was suspended in resuspension buffer (with $0.1 \%$ Tween-20, $0.01 \%$ digitonin and $0.1 \%$ NP40).

For snATAC-seq, the isolation of nuclei was done according to $10 \times$ Genomics protocol CG000212 (Rev A) with minor modification. For both strains midbrain sections from two hemibrains of the same mouse were processed separately and pooled at the analysis level. In brief, frozen midbrain sections were minced in a Dounce Tissue Grinder (Sigma, D8939-1SET) with A pestle 10 times following B pestle $\sim 20$ times in $500 \mathrm{uL}$ chilled lysis buffer [10 mM Tris- $\mathrm{HCl}$ (pH7.4), $10 \mathrm{mM} \mathrm{NaCl}, 3 \mathrm{mM}$ $\mathrm{MgCl}_{2}, 0.1 \%$ Tween-20, 0.1\% NP40, 0.01\% Digitonin, 1\% BSA]. The homogenized lysate was incubated on ice for $5 \mathrm{~min}$, following pipette mixing $10 \times$ and incubated again on ice for $10 \mathrm{~min}$. Chilled Wash Buffer $(500 \mu \mathrm{L})[10 \mathrm{mM}$ Tris-HCl (pH 7.4), $10 \mathrm{mM} \mathrm{Nacl} 3 \mathrm{mM} \mathrm{MgCl}$, $1 \%$ BSA, $0.1 \%$ Tween-20] was added to the lysed cells and pipetted mix $5 \times$. The lysate was layered on $3 \mathrm{~mL}$ sucrose cushion [1.8 $\mathrm{M}$ sucrose, $3 \mathrm{mM} \mathrm{Mg(Ac)2,} 10 \mathrm{mM}$ Tris $\mathrm{pH}$ 8.0, $0.167 \mathrm{mM} \beta$-mercaptoethanol], following ultra-centrifugation $30,000 \times g$ for $1 \mathrm{~h}$ (Rotor:Beckman Coulter, MLS50) at $4{ }^{\circ} \mathrm{C}$. After centrifugation, the supernatant was discarded and the nuclei pellet was suspended in Nuclei Buffer $(10 \times$ PN: 2000153$)$ provided by $10 \times$ Genomics Chromium Single Cell ATAC Reagent Kit. The nuclei suspension was passed through a $40 \mu \mathrm{m}$ Flowmi Cell Strainer (Sigma, BAH136800040-50EA).

\section{Bulk ATAC-seq}

Tagmentation of a midbrain sample from a C57BL/6J mouse was done based on the OMNI-ATAC supplementary protocol 1 [72] with minor changes. Briefly, 25,000 nuclei were resuspended in $50 \mu \mathrm{L}$ resuspension buffer (with $0.1 \%$ Tween-20, $0.01 \%$ digitonin and $0.1 \%$ NP40) and lysed for $3 \mathrm{~min}$ on ice. After washing in $1 \mathrm{~mL}$ resuspension buffer (1\% Tween-20) samples were centrifuged for $10 \mathrm{~min}$ at $500 \times g$ at $4{ }^{\circ} \mathrm{C}$ and supernatant carefully removed. Pellets were resuspended in $25 \mu \mathrm{L}$ tagmentation mix (Tagment DNA buffer from Illumina, \#15027866) containing $2.5 \mu \mathrm{L}$ Tagment DNA Enzyme (Illumina \#15027865) and incubated for $45 \mathrm{~min}$ at $37^{\circ} \mathrm{C}$ and $1000 \mathrm{rpm}$ in Eppendorf ThermoMixer. Tagmented 
chromatin was isolated using Zymo Research DNA Clean \& Concentrator kit (ZymoResearch ZY-D4013) and eluted in $21 \mu \mathrm{L}$ elution buffer. Library pre-amplification was done for 5 cycles using primers Ad1 and Ad2.16 [10]. Five additional cycles of library amplification were done as determined by qPCR [72]. Library cleanup was done using Zymo Research DNA Clean \& Concentrator kit followed by AMPure XP bead (Beckman Coulter \#A63880) size selection. A first bead incubation using $0.55 \times$ volume of beads removes large fragments. After separation of beads on magnetic stand, supernatant was transferred to a fresh tube and incubated for $5 \mathrm{~min}$ in $1.5 \times$ volumes of beads. After washing with $80 \%$ ethanol, beads were resuspended in $20 \mu \mathrm{L}$ elution buffer. After separation on magnetic stand eluate was transferred to a fresh tube. Library quality was assessed using Agilent DNA High sensitivity Bioanalyzer chip (Agilent \#5067-4626).

\section{Bulk ATAC-seq data analysis}

The sequencing of ATAC-seq libraries was done at the sequencing platform in the Luxembourg Centre for Systems Biomedicine (LCSB) of the University of Luxembourg. The paired-end, unstranded library sequencing was performed using Illumina NextSeq 500/550 75 cycles High Output Kit. Raw FASTQ files and BAM files were processed as described in the "Methods" section for ChIP-seq. After processing of the BAM files, the peaks were called by Genrich (https://github.com/jsh58/Genri ch) with parameters "-r -m 30 -j" to remove PCR duplicates and include only reads with mapping quality of at least 30. Footprints were called by HINT-ATAC [73] with default parameters. Raw FASTQ files were deposited in ArrayExpress with the accession number E-MTAB-8333.

Single-nuclei ATAC-seq library preparation and sequencing The single-nuclei ATAC-seq was performed according to Chrominum Single Cell ATAC Reagent Kits User Guide (CG000168 RevB) with Chromium Single Cell E Chip Kit $(10 \times, 100086)$, Chromium Single Cell ATAC Library \& Gel Bead Kit $(10 \times, 1000111)$, Chromium Single Cell ATAC Gel Bead Kit $(10 \times, 1000085)$, Chromium Single Cell ATAC Library Kit $(10 \times 100087)$, Chromium i7 Multiplex Kit N Set (1000084), Dynabeads MyOne Silane (2000048). In brief, nuclei suspension was loaded with a targeted recovery rate of 10,000 nuclei per sample. snATAC-seq libraries quality were assessed using Agilent DNA High sensitivity Bioanalyzer chip (Agilent \#5067-4626) and further sequenced on a 150 cycles High Output Kit using Illumina NextSeq ${ }^{\mathrm{TM}} 500$ with targeted sequencing depth of 25,000 read pairs per nucleus. Raw FASTQ files were deposited in ArrayExpress with the Accession number E-MTAB-9225.

\section{Single-nuclei ATAC-seq analysis pipeline Cell ranger}

The alignment and filtering were done according to $10 \times$ running pipelines. In brief, the fastq files was generated from Illumina sequencer's base call files, which were later used as inputs to align (MAPQ $>30)$, filter barcode multiplets and generate accessibility counts for each cell in a single library. The technical replicates for each mouse strain were aggregated to create a single peak-barcode matrix. Each unique fragment is associated with a single cell barcode.

\section{Clustering}

The snATAC-seq downstream analysis was performed by Signac (version: 0.2 .4 ) in R. The gene activity matrix was calculated with reads in gene body and $2 \mathrm{~kb}$ upstream of TSS as a proxy for gene expression. Nuclei with counts less than 5000 were filtered out. The dimensionality was calculated with latent semantic index (LSI) on peaks with at least 100 reads across all cells, which was used as input to generate UMAP graphs.

\section{Cluster annotation}

The annotation of snATAC-seq clusters took use of the existing scRNA-seq data on midbrains from adult 3-month-old C57BL/6N mice. The anchors were found between the gene activity matrix of snATAC-seq and the top 5000 variable features of scRNA-seq. The cell labels were transferred from the scRNA-seq to the snATAC-seq with normalization on anchor weights calculated from the LSI dimensional reduction, resulting in 10,347 nuclei of C57BL/6J and 10,368 nuclei of A/J annotated.

\section{scRNA-seq data analysis pipeline Data processing}

The digital gene expression (DGE) and cell annotation for midbrains of 3-month-old $\mathrm{C} 57 \mathrm{BL} / 6 \mathrm{~N}$ mice were downloaded from DropViz. The data analysis was performed by Seurat (version: 3.1.4) in R. Only cells with feature counts between 400 to 7000 and being single or well-curated were used in downstream analysis, resulting in 19,967 cells in total. The DGE was natural-log transformed and normalized to mitochondrial read counts. The dimensional reduction was done with UMAP. The clusters were annotated with existing annotation from DropViz.

\section{Selection of cell-type-identity genes}

100 cells were randomly selected from each cell cluster. DGE from each cell type was constructed according to 
corresponding barcodes. The 85th percentile expression for each gene was calculated on the selected cells. The criteria for cell-type-identity genes was defined as: For a particular gene, $60 \%$ of cells in a cell type have expression larger than the gene specific 85th percentile; while at most $40 \%$ of cells in all other cell types have expression larger than the 85th percentile. This process were repeated for 100 times. Genes that appeared more than 30 times out of 100 were defined as the cell-typeidentity genes.

\section{Defining differential peaks}

Differential peaks were defined for each cell type using Wilcoxon rank-sum test (implemented in $\mathrm{R}$ package "presto" 1.0.0). The binary peak count matrix from scATAC-seq was normalized by the number of peaks presented in each cell. Then the differential peak analysis was performed with presto on the normalized peak count matrix. Peaks with FDR $<0.05$ and $\log$ foldchange $(\log F C)$ in top $1 \%$ are defined as differential peaks.

\section{Motif enrichment analysis}

\section{Generating cell-type-specific bam files}

The cell-type-specific bamfiles were generated by samtools. The barcodes from each replicate of a strain in bamfile was relabelled to avoid barcode collapse. After relabelling, the bamfiles from replicates of a sample were merged. The bamfile for each cell type were subtracted based on corresponding barcodes.

\section{Peak calling}

The peak calling was done by MACS2 (2.1.2) [74] with custom cutoff on $p$ values according to cutoff analysis with parameters 'macs2 callpeak -cutoff-analysis'. The ideal cutoff was chosen based on that the selected $p$ value would not lead to exponential increase of peak numbers.

\section{Motif enrichment analysis}

The motif enrichment analysis was performed by HOMER (4.11.1) [75] with parameters 'findMotifsGenome.pl -size given -mask'.

\section{Chromatin immunoprecipitation (ChIP)}

ChIP was performed on the dissected snap frozen mouse ventral midbrain tissue from two independent mice per strain as previously described [20]. Each reaction had $10-14 \mu \mathrm{g}$ of chromatin and $10 \%$ aliquot was used as input DNA. Immunoprecipitation was performed overnight with $5 \mu \mathrm{L}$ of H3K27ac antibody (Abcam, ab4729), at $4{ }^{\circ} \mathrm{C}$ with rotation.

\section{ChIP-seq data analysis}

The sequencing of the chromatin samples was done at the sequencing platform in the LCSB of the University of Luxembourg. The single-end, unstranded sequencing with read length of $75 \mathrm{bp}$ was performed with Illumina NextSeq 500 machine. FastQC (v0.11.5) was used for raw reads quality assessment [76]. Generation of BAM files, including steps of adapter removal, mapping and duplicate marking, were done with PALEOMIX pipeline (v1.2.12) [77], followed by mapping with BWA (v.0.7.16a) [78]. Backtrack algorithm applied the quality offset of Phred score to 33. Duplicate reads were marked and the mouse reference genome, GRCm38.p5 (mm10, patch 5) was downloaded from GENCODE (https://www.gencodegen es.org/). Finally, validation of the Bam files was done using Picard (v2.10.9) [79]. Raw FASTQ files were deposited in ArrayExpress with the Accession number E-MTAB-8333.

The H3K27ac ChIP-seq peaks, enhancers and superenhancers, were called by HOMER [75] with default parameters. The signal normalization in pairwise comparison was done by THOR (v0.10.2) [41], with TMM normalization and adjusted $p$ value cutoff 0.01 .

\section{Supplementary Information}

The online version contains supplementary material available at https://doi. org/10.1186/s13072-021-00418-3.

Additional file 1: Figure S1. snATAC-seq on ventral midbrains of C57BL/6J and A/J revealed cell-type-specific chromatin accessibility. Figure S2. H3K27ac ChIP-seq and ATAC-seq correlating with gene expression. Figure S3. Differential peaks can reveal strain-specific TFs.

Additional file 2: Table S1. Cell-type composition in ventral midbrains of C57BL/6J and A/J in snATACseq. Cell types and identified cell numbers are indicated for both strains.

Additional file 3: Table S2. Cell-type-identity genes defined from existing scRNA-seq. The official gene symbols for the identified cell identity genes are listed under each respective cell type.

Additional file 4: Table S3. Enrichment analysis on the cell-type-identity genes defined from existing scRNA-seq. The GO enrichment results for the cell identity genes for each cell type are provided. The enrichment analysis was performed using Enrichr and GO term identifiers, enrichment $p$ values, scores, and identified gene names are provided for each GO term.

Additional file 5: Table S4. Cell-type-identity peaks defined by associating cell-type-specific peaks to the regulatory regions of cell-type-identity genes. The chromosome coordinates for all cell identity peaks are provided for each cell type.

Additional file 6: Table S5. Putative regulatory variants. The chromosome coordinates and major/alternative alleles are reported for the 3909 variants locating within TF footprints in active enhancers and designated as putative regulatory variants.

Additional file 7: Table S6. Differentially expressed genes from ventral midbrain bulk RNA-seq between C57BL/6J and A/J. Each strain has 12 replicates ( 6 males and 6 females). The differential genes are defined as padj $<0.05$.

Additional file 8: Table S7. H3K27ac differential peaks between C57BL/6J and $\mathrm{A} / \mathrm{J}$. The chromosomecoordinates of the peaks and $p$-values of sign3a I difference between the strains are indicated for each differential peak $\left(p<1 \times 10^{-18}\right)$. 
Additional file 9: Table S8. Predicted upstream regulators between $\mathrm{C} 57 \mathrm{BL} / 6 \mathrm{~J}$ and $\mathrm{A} / \mathrm{J}$. The predicted upstream regulators are fetched from IPA based on differentially expressed genes from midbrain bulk RNA-seq between C57BL/6J and A/J (padj < 0.05, log2FC > 1).

\section{Acknowledgements}

We would like to thank Drs. Aurélien Ginolhac and Anthoula Gaigneaux for their support with bioinformatic analysis, Dr. Djalil Coowar (Animal Facility of University of Luxembourg) for help with breeding of experimental mice, Dr. Rashi Halder at LCSB sequencing facility for high-throughput sequencing, and Sergio Helgueta for help with nuclei isolation. The computational analysis presented in this paper were carried out using the HPC facilities of the University of Luxembourg.

\section{Authors' contributions}

$Y G, M B$, and $L S$ conceived the project. $Y G, K G, J O, A S$, TS, and $L S$ designed the experiments. YG and KG performed snATAC-seq. YG and JO performed bulk ATAC-seq and YG performed ChIP-seq experiments. MHT and PG prepared mouse tissues. YG performed all bioinformatic analysis with help from TS and LS. YG and LS analysed the results. MB, TS, and LS provided funding. YG and LS wrote the manuscript. All authors read and approved the final manuscript.

\section{Funding}

LS and MB would like to thank the Luxembourg National Research Fund (FNR) for the support (FNR CORE C15/BM/10406131 grant). LS and JO would like to thank Fondation du Pélican de Mie et Pierre Hippert-Faber and Luxembourg Rotary Foundation for funding. MHT, PG, and MB wish to thank Prof. M. Mittelbronn, who is funded by FNR PEARL P16/BM/11192868 grant, for support.

\section{Availability of data and materials}

Raw FASTQ files were deposited in ArrayExpress with the accession number E-MTAB-8333 for bulk levels analysis and E-MTAB-9225 for single cell data. The snATAC-seq tracks are available at the UCSC Genome Browser (http:// genome-euro.ucsc.edu/cgi-bin/hgTracks?hubUrl=https://biostat2.uni.lu/ygui/ hub.txt\&genome $=\mathrm{mm} 10$ ). The scripts for data analysis can be accessed here: https://github.com/sysbiolux/Gui-et-al.

\section{Declarations}

Ethics approval and consent to participate

The animal protocol was approved by the Animal Experimentation Ethics Committee (AEEC) according to the national guidelines of the animal welfare law in Luxembourg (Règlement grand-ducal adopted on January 11th, 2013).

\section{Consent for publication}

Not applicable.

\section{Competing interests}

The authors declare that they have no competing interests.

\section{Author details}

${ }^{1}$ Department of Life Sciences and Medicine (DLSM), University of Luxembourg, Belvaux, Luxembourg. ' Luxembourg Centre for Systems Biomedicine (LCSB), University of Luxembourg, Belvaux, Luxembourg.

Received: 15 June 2021 Accepted: 24 August 2021

Published online: 09 September 2021

\section{References}

1. Vogt Weisenhorn DM, Giesert F, Wurst W. Diversity matters-heterogeneity of dopaminergic neurons in the ventral mesencephalon and its relation to Parkinson's Disease. J Neurochem. 2016;139(Suppl 1):8-26.

2. Klein C, Westenberger A. Genetics of Parkinson's disease. Cold Spring Harb Perspect Med. 2012;2(1):a008888.
3. Li Z, Chen J, Yu H, He L, Xu Y, Zhang D, et al. Genome-wide association analysis identifies 30 new susceptibility loci for schizophrenia. Nat Genet. 2017:49(11):1576-83.

4. Nalls MA, Blauwendraat C, Vallerga CL, Heilbron K, Bandres-Ciga S, Chang $D$, et al. Identification of novel risk loci, causal insights, and heritable risk for Parkinson's disease: a meta-analysis of genome-wide association studies. Lancet Neurol. 2019;18(12):1091-102.

5. Williams HJ, Owen MJ, O'Donovan MC. New findings from genetic association studies of schizophrenia. J Hum Genet. 2009;54(1):9-14.

6. Gantz SC, Ford CP, Morikawa H, Williams JT. The evolving understanding of dopamine neurons in the substantia nigra and ventral tegmental area. Annu Rev Physiol. 2018;80(1):219-41.

7. Korotkova TM, Ponomarenko AA, Brown RE, Haas HL. Functional diversity of ventral midbrain dopamine and GABAergic neurons. Mol Neurobiol. 2004;29(3):243-59.

8. Saunders A, Macosko EZ, Wysoker A, Goldman M, Krienen FM, de Rivera $\mathrm{H}$, et al. Molecular diversity and specializations among the cells of the adult mouse brain. Cell. 2018;174(4):1015-1030.e16.

9. Atlasi Y, Stunnenberg HG. The interplay of epigenetic marks during stem cell differentiation and development. Nat Rev Genet. 2017;18(11):643-58.

10. Buenrostro J, Wu B, Chang H, Greenleaf W. ATAC-seq: a method for assaying chromatin accessibility genome-wide. Curr Protoc Mol Biol Ed Frederick M Ausubel Al. 2015;109:21.29.1-21.29.9.

11. Cusanovich DA, Hill AJ, Aghamirzaie D, Daza RM, Pliner HA, Berletch JB, et al. A single-cell atlas of in vivo mammalian chromatin accessibility. Cell. 2018;174(5):1309-1324.e18.

12. Lake BB, Chen S, Sos BC, Fan J, Kaeser GE, Yung YC, et al. Integrative single-cell analysis of transcriptional and epigenetic states in the human adult brain. Nat Biotechnol. 2018;36(1):70-80.

13. Preiss S, Fang R, Huang H, Zhao Y, Raviram R, Gorkin DU, et al. Singlenucleus analysis of accessible chromatin in developing mouse forebrain reveals cell-type-specific transcriptional regulation. Nat Neurosci. 2018;21(3):432-9.

14. Sinnamon JR, Torkenczy KA, Linhoff MW, Vitak SA, Mulqueen RM, Pliner HA, et al. The accessible chromatin landscape of the murine hippocampus at single-cell resolution. Genome Res. 2019;29(5):857-69.

15. Auton A, Abecasis GR, Altshuler DM, Durbin RM, Abecasis GR, Bentley $D R$, et al. A global reference for human genetic variation. Nature. 2015;526(7571):68-74.

16. Maurano MT, Humbert R, Rynes E, Thurman RE, Haugen E, Wang H, et al. Systematic localization of common disease-associated variation in regulatory DNA. Science. 2012;337(6099):1190-5.

17. Deplancke B, Alpern D, Gardeux V. The genetics of transcription factor DNA binding variation. Cell. 2016;166(3):538-54.

18. Moy SS, Nadler JJ, Young NB, Perez A, Holloway LP, Barbaro RP, et al. Mouse behavioral tasks relevant to autism: Phenotypes of 10 inbred strains. Behav Brain Res. 2007;176(1):4-20.

19. Thifault S, Lalonde R, Sanon N, Hamet P. Comparisons between C57BL/6J and $\mathrm{A} / \mathrm{J}$ mice in motor activity and coordination, hole-poking, and spatial learning. Brain Res Bull. 2002;58(2):213-8.

20. Gui Y, Thomas MH, Garcia P, Karout M, Halder R, Michelucci A, et al. Pituitary tumor transforming gene 1 orchestrates gene regulatory variation in mouse ventral midbrain during aging. Front Genet. 2020. https://doi.org/ 10.3389/fgene.2020.566734.

21. Rada-Iglesias A, Bajpai R, Swigut T, Brugmann SA, Flynn RA, Wysocka J. A unique chromatin signature uncovers early developmental enhancers in humans. Nature. 2011;470(7333):279-83.

22. Siersbæk R, Madsen JGS, Javierre BM, Nielsen R, Bagge EK, Cairns J, et al. Dynamic rewiring of promoter-anchored chromatin loops during adipocyte differentiation. Mol Cell. 2017;66(3):420-435.e5.

23. Butler A, Hoffman P, Smibert P, Papalexi E, Satija R. Integrating single-cell transcriptomic data across different conditions, technologies, and species. Nat Biotechnol. 2018. https://www.nature.com/articles/nbt.4096. Accessed 3 Apr 2018.

24. Satija R, Farrell JA, Gennert D, Schier AF, Regev A. Spatial reconstruction of single-cell gene expression data. Nat Biotechnol. 2015;33(5):495-502.

25. Bennett ML, Bennett FC, Liddelow SA, Ajami B, Zamanian JL, Fernhoff NB, et al. New tools for studying microglia in the mouse and human CNS. Proc Natl Acad Sci USA. 2016;113(12):E1738-46. 
26. Cahoy JD, Emery B, Kaushal A, Foo LC, Zamanian JL, Christopherson KS, et al. A transcriptome database for astrocytes, neurons, and oligodendrocytes: a new resource for understanding brain development and function. J Neurosci. 2008;28(1):264-78.

27. Lu QR, Sun T, Zhu Z, Ma N, Garcia M, Stiles CD, et al. Common developmental requirement for olig function indicates a motor neuron/oligodendrocyte connection. Cell. 2002;109(1):75-86.

28. Mei F, Wang H, Liu S, Niu J, Wang L, He Y, et al. Stage-specific deletion of Olig2 conveys opposing functions on differentiation and maturation of oligodendrocytes. J Neurosci. 2013;33(19):8454-62.

29. McLean CY, Bristor D, Hiller M, Clarke SL, Schaar BT, Lowe CB, et al. GREAT improves functional interpretation of cis-regulatory regions. Nat Biotechnol. 2010;28(5):495-501.

30. Ginhoux F, Greter M, Leboeuf M, Nandi S, See P, Gokhan S, et al. Fate mapping analysis reveals that adult microglia derive from primitive macrophages. Science. 2010;330(6005):841-5.

31. Stolt CC, Lommes P, Sock E, Chaboissier M-C, Schedl A, Wegner M. The Sox9 transcription factor determines glial fate choice in the developing spinal cord. Genes Dev. 2003;17(13):1677-89.

32. Gosselin D, Skola D, Coufal NG, Holtman IR, Schlachetzki JCM, Sajti E, et al. An environment-dependent transcriptional network specifies human microglia identity. Science. 2017. https://science.sciencemag.org/conte nt/early/2017/05/24/science.aal3222. Accessed 22 May 2020

33. Kierdorf K, Erny D, Goldmann T, Sander V, Schulz C, Perdiguero EG, et al. Microglia emerge from erythromyeloid precursors via Pu.1- and Irf8dependent pathways. Nat Neurosci. 2013;16(3):273-80.

34. Liu M, Zhang L, Marsboom G, Jambusaria A, Xiong S, Toth PT, et al. Sox17 is required for endothelial regeneration following inflammation-induced vascular injury. Nat Commun. 2019;10(1):2126.

35. Stolt CC, Rehberg S, Ader M, Lommes P, Riethmacher D, Schachner $\mathrm{M}$, et al. Terminal differentiation of myelin-forming oligodendrocytes depends on the transcription factor Sox10. Genes Dev. 2002;16(2):165-70.

36. Deneen B, Ho R, Lukaszewicz A, Hochstim CJ, Gronostajski RM, Anderson $D J$. The transcription factor NFIA controls the onset of gliogenesis in the developing spinal cord. Neuron. 2006;52(6):953-68.

37. Wilczynska KM, Singh SK, Adams B, Bryan L, Rao RR, Valerie K, et al. Nuclear factor $\mathrm{I}$ isoforms regulate gene expression during the differentiation of human neural progenitors to astrocytes. Stem Cells. 2009;27(5):1173-81.

38. Matcovitch-Natan O, Winter DR, Giladi A, Aguilar SV, Spinrad A, Sarrazin $S$, et al. Microglia development follows a stepwise program to regulate brain homeostasis. Science. 2016;353(6301). https://science.sciencemag. org/content/353/6301/aad8670. Accessed 22 May 2020.

39. Sugiaman-Trapman D, Vitezic M, Jouhilahti E-M, Mathelier A, Lauter G, Misra S, et al. Characterization of the human RFX transcription factor family by regulatory and target gene analysis. BMC Genomics. 2018;19(1):181.

40. Pierce SE, Tyson T, Booms A, Prahl J, Coetzee GA. Parkinson's disease genetic risk in a midbrain neuronal cell line. Neurobiol Dis. 2018;114:53-64.

41. Allhoff M, Seré K, Pires JF, Zenke M, Costa IG. Differential peak calling of ChIP-seq signals with replicates with THOR. Nucleic Acids Res. 2016:44(20):e153.

42. Morales M, Margolis EB. Ventral tegmental area: cellular heterogeneity, connectivity and behaviour. Nat Rev Neurosci. 2017;18(2):73-85.

43. Ponder CA, Kliethermes CL, Drew MR, Muller J, Das K, Risbrough VB, et al. Selection for contextual fear conditioning affects anxiety-like behaviors and gene expression. Genes Brain Behav. 2007;6(8):736-49.

44. Portugal GS, Wilkinson DS, Kenney JW, Sullivan C, Gould TJ. Straindependent effects of acute, chronic, and withdrawal from chronic nicotine on fear conditioning. Behav Genet. 2012;42(1):133-50.

45. Yoneyama N, Crabbe JC, Ford MM, Murillo A, Finn DA. Voluntary ethanol consumption in 22 inbred mouse strains. Alcohol. 2008;42(3):149-60.

46. Nusse R, Clevers H. Wnt/ $\beta$-catenin signaling, disease, and emerging therapeutic modalities. Cell. 2017;169(6):985-99.

47. Mao CD, Byers SW. Cell-context dependent TCF/LEF expression and function: alternative tales of repression, de-repression and activation potentials. Crit Rev Eukaryot Gene Expr. 2011;21(3):207-36.

48. Liu K, Pan C, Kuhn A, Nievergelt AP, Fantner GE, Milenkovic O, et al. Detecting topological variations of DNA at single-molecule level. Nat Commun. 2019;10(1):3.
49. Wu TP, Wang T, Seetin MG, Lai Y, Zhu S, Lin K, et al. DNA methylation on $\mathrm{N} 6$-adenine in mammalian embryonic stem cells. Nature. 2016;532(7599):329-33.

50. Arnold CD, Gerlach D, Stelzer C, Boryń ŁM, Rath M, Stark A. Genome-wide quantitative enhancer activity maps identified by STARR-seq. Science. 2013;339(6123):1074-7.

51. McKay DJ, Lieb JD. A common set of DNA regulatory elements shapes Drosophila appendages. Dev Cell. 2013;27(3):306-18.

52. Lara-Astiaso D, Weiner A, Lorenzo-Vivas E, Zaretsky I, Jaitin DA, David $E$, et al. Chromatin state dynamics during blood formation. Science. 2014;345(6199):943-9.

53. Pálfy M, Schulze G, Valen E, Vastenhouw NL. Chromatin accessibility established by Pou5f3, Sox19b and Nanog primes genes for activity during zebrafish genome activation. PLoS Genet. 2020;16(1):e1008546.

54. McGary KL, Park TJ, Woods JO, Cha HJ, Wallingford JB, Marcotte EM. Systematic discovery of nonobvious human disease models through orthologous phenotypes. Proc Natl Acad Sci. 2010;107(14):6544-9.

55. Lodato MA, Ng CW, Wamstad JA, Cheng AW, Thai KK, Fraenkel E, et al. SOX2 co-occupies distal enhancer elements with distinct POU factors in ESCs and NPCs to specify cell state. PLOS Genet. 2013;9(2):e1003288.

56. Fay JC, McCullough HL, Sniegowski PD, Eisen MB. Population genetic variation in gene expression is associated with phenotypic variation in Saccharomyces cerevisiae. Genome Biol. 2004;5(4):R26.

57. Keane TM, Goodstadt L, Danecek P, White MA, Wong K, Yalcin B, et al. Mouse genomic variation and its effect on phenotypes and gene regulation. Nature. 2011;477(7364):289-94.

58. Massouras A, Waszak SM, Albarca-Aguilera M, Hens K, Holcombe W, Ayroles JF, et al. Genomic variation and its impact on gene expression in Drosophila melanogaster. PLOS Genet. 2012;8(11):e1003055.

59. Storey JD, Madeoy J, Strout JL, Wurfel M, Ronald J, Akey JM. Gene-expression variation within and among human populations. Am J Hum Genet. 2007;80(3):502-9.

60. Cao J, Cusanovich DA, Ramani V, Aghamirzaie D, Pliner HA, Hill AJ, et al. Joint profiling of chromatin accessibility and gene expression in thousands of single cells. Science. 2018;361(6409):1380-5.

61. Ge D, Fellay J, Thompson AJ, Simon JS, Shianna KV, Urban TJ, et al. Genetic variation in IL28B predicts hepatitis $C$ treatment-induced viral clearance. Nature. 2009:461(7262):399-401.

62. Grundberg E, Small KS, Hedman ÅK, Nica AC, Buil A, Keildson S, et al. Mapping cis- and trans -regulatory effects across multiple tissues in twins. Nat Genet. 2012;44(10):1084-9.

63. Ohnmacht J, May P, Sinkkonen L, Krüger R. Missing heritability in Parkinson's disease: the emerging role of non-coding genetic variation. J Neural Transm. 2020. https://doi.org/10.1007/s00702-020-02184-0.

64. Crowley JJ, Zhabotynsky V, Sun W, Huang S, Pakatci IK, Kim Y, et al. Analyses of allele-specific gene expression in highly divergent mouse crosses identifies pervasive allelic imbalance. Nat Genet. 2015;47(4):353-60.

65. Nott A, Holtman IR, Coufal NG, Schlachetzki JCM, Yu M, Hu R, et al. Brain cell type-specific enhancer-promoter interactome maps and diseaserisk association. Science. 2019;366(6469):1134-9.

66. Bogue MA, Philip VM, Walton DO, Grubb SC, Dunn MH, Kolishovski $\mathrm{G}$, et al. Mouse Phenome Database: a data repository and analysis suite for curated primary mouse phenotype data. Nucleic Acids Res. 2020;48(D1):D716-23.

67. Albert FW, Bloom JS, Siegel J, Day L, Kruglyak L. Genetics of transregulatory variation in gene expression. Wittkopp PJ, editor. eLife. 2018;7:e35471.

68. Liu X, Li Yl, Pritchard JK. Trans effects on gene expression can drive omnigenic inheritance. Cell. 2019;177(4):1022-1034.e6.

69. Bem J, Brożko N, Chakraborty C, Lipiec MA, Koziński K, Nagalski A, et al. Wnt/ $\beta$-catenin signaling in brain development and mental disorders: keeping TCF7L2 in mind. FEBS Lett. 2019;593(13):1654-74.

70. Winham SJ, Cuellar-Barboza AB, Oliveros A, McElroy SL, Crow S, Colby $C$, et al. Genome-wide association study of bipolar disorder accounting for effect of body mass index identifies a new risk allele in TCF7L2. Mol Psychiatry. 2014;19(9):1010-6.

71. Sittig LJ, Carbonetto P, Engel KA, Krauss KS, Barrios-Camacho CM, Palmer AA. Genetic background limits generalizability of genotype-phenotype relationships. Neuron. 2016;91(6):1253-9.

72. Corces MR, Trevino AE, Hamilton EG, Greenside PG, Sinnott-Armstrong NA, Vesuna S, et al. An improved ATAC-seq protocol reduces 
background and enables interrogation of frozen tissues. Nat Methods 2017;14(10):959-62

73. Li Z, Schulz MH, Look T, Begemann M, Zenke M, Costa IG. Identification of transcription factor binding sites using ATAC-seq. Genome Biol. 2019;20(1):45

74. Feng J, Liu T, Qin B, Zhang Y, Liu XS. Identifying ChIP-seq enrichment using MACS. Nat Protoc. 2012;7(9):1728-40.

75. Heinz S, Benner C, Spann N, Bertolino E, Lin YC, Laslo P, et al. Simple combinations of lineage-determining transcription factors prime cisregulatory elements required for macrophage and $B$ cell identities. $\mathrm{Mol}$ Cell. 2010;38(4):576-89.

76. Andrews S. FastQC: a quality control tool for high throughput sequence data. 2010.

77. Schubert M, Ermini L, Sarkissian CD, Jónsson H, Ginolhac A, Schaefer R, et al. Characterization of ancient and modern genomes by SNP detection and phylogenomic and metagenomic analysis using PALEOMIX. Nat Protoc. 2014;9(5):1056-82.

78. Li H, Handsaker B, Wysoker A, Fennell T, Ruan J, Homer N, et al. The sequence alignment/map format and SAMtools. Bioinformatics. 2009;25(16):2078-9.

79. Adams MD, Celniker SE, Holt RA, Evans CA, Gocayne JD, Amanatides PG, et al. The Genome Sequence of Drosophila melanogaster. Science. 2000;287(5461):2185-95.

\section{Publisher's Note}

Springer Nature remains neutral with regard to jurisdictional claims in published maps and institutional affiliations.
Ready to submit your research? Choose BMC and benefit from:

- fast, convenient online submission

- thorough peer review by experienced researchers in your field

- rapid publication on acceptance

- support for research data, including large and complex data types

- gold Open Access which fosters wider collaboration and increased citations

- maximum visibility for your research: over 100M website views per year

At BMC, research is always in progress.

Learn more biomedcentral.com/submissions 